\title{
Electrorheology of a dilute emulsion of surfactant-covered drops
}

\author{
Antarip Poddar ${ }^{1}$, Shubhadeep Mandal ${ }^{1,2}$, Aditya Bandopadhyay*1, \\ and Suman Chakraborty ${ }^{\dagger 1}$ \\ ${ }^{1}$ Department of Mechanical Engineering, Indian Institute of Technology Kharagpur, Kharagpur, \\ West Bengal - 721302, India \\ ${ }^{2}$ Max Planck Institute for Dynamics and Self-Organization, Am Fassberg 17, D-37077 Göttingen, \\ Germany
}

\begin{abstract}
The effects of surfactant coating on a deformable viscous drop under the combined action of a shear flow and a uniform electric field, are investigated by solving the coupled equations of electrostatics, fluid flow and surfactant transport. Employing a comprehensive three-dimensional solution technique, the non-Newtonian shearing response of the bulk emulsion is analyzed in the dilute suspension regime. The present results reveal that the surfactant non-uniformity creates significant alterations in the flow disturbance around the drop, thereby influencing the viscous dissipation from the flowing emulsion. This, in effect, triggers changes in the bulk shear viscosity. It is striking to observe that the balance between electrical and hydrodynamic stresses is affected in such a way that surface tension gradient on the drop surface vanishes for some specific shear rates and the corresponding effective change in the bulk viscosity becomes negligible too. This critical condition hugely depends on the electrical permittivity and conductivity ratios of the two fluids and orientation of the applied electric field. Also the physical mechanisms of charge convection of surface deformation play their role in determining this critical shear rate. The charge convection instigated shear thinning or shear thickening behavior of the emulsion gets reversed due to a coupled interaction of the charge convection and Marangoni stress. In addition, the electrically created anisotropic normal stresses in the bulk rheology, get reduced due to the presence of surfactants, especially when the drop viscosity is much lesser than the continuous fluid. A thorough description of the drop-level flow physics and its connection to the bulk rheology of a dilute emulsion, may provide a fundamental understanding of a more complex emulsion system.
\end{abstract}

*Email: aditya@mech.iitkgp.ernet.in

†Email: suman@mech.iitkgp.ernet.in 


\section{Introduction}

An immiscible dispersion of liquid drops in another liquid medium is ubiquitous in diverse domains of practical interest, ranging from food and material processing, pharmaceutical industries to petroleum refinery (Eow \& Ghadiri, 2002, Barnes, 1994). These dispersions, commonly known as emulsions, can also be generated in the controlled environments of the state-of-the art microfluidic techniques (Anna et al., 2003; Haliburton et al., 2017). Variations in the microstructure and morphology of the individual drops in the dispersed phase may lead to drastic alterations in the bulk physicochemical properties of the emulsion (Tucker \& Moldenaers, 2002, Puyvelde et al., 2001; Lequeux, 1998). In addition, the application of external stresses has proved to be immensely useful in modifying the distribution, deformation and motion of the individual constituents of the emulsion.

In suspension mechanics, the bulk scale description of the dispersion is often provided by considering a continuous effective fluid. Now the disturbance in flow due to the stresses exerted on the drops, affects the dissipation of energy by the continuous fluid. Consequently the stress-response of the bulk mixture deviates from that of a Newtonian fluid (Guazzelli \& Morris, 2011). Taylor (1932) was the first to theoretically predict the contribution of dropphase in increasing the macroscale viscosity of a dilute emulsion under an imposed linear flow. Later, it was found that the deformable nature of the suspended drops can not only impart an elastic nature to the emulsion rheology (Schowalter et al., 1968) but also makes it exhibit a shear-thinning property (Barths-Biesel \& Acrivos, 1973). The nature of drop break-up and deformation under different linear flow fields was also characterized (Rallison, 1984, Bentley \& Leal, 1986). It was observed that under a two dimensional linear shear flow, the drop makes an angle of $\pi / 4$ with the imposed flow direction and deforms into an ellipsoidal shape when the of surface tension is high compared to the flow strength. Further, the rotational component of the shear flow tries to reorient the drop.

One of the most promising techniques regarding the manipulation of rheological properties of an emulsion is the application of electric field (Pan \& McKinley, 1997; Ha \& Yang, 2000; Na et al., 2009). Electrohydrodynamic effects can deform a drop in an oblate or prolate spheroidal shape, depending on the combination of the electrical properties of the dispersed and continuous phase (Taylor, 1966; Xu et al., 2006; Esmaeeli \& Sharifi, 2011; Thaokar, 2012; Lanauze et al., 2015). In the case of leaky dielectric fluids, the electric field also induces a tangential flow around the drop (Saville, 1997). The electrohydrodynamic flow and drop deformation get further affected by the presence of finite charge convection at the interface (Feng, 1999; Xu \& Homsy, 2006; Yariv \& Almog, 2016; Das \& Saintillan, 2016). The significance of a tilted electric field configuration in breaking the fore-aft symmetry of a sedimenting drop was experimentally shown by Bandopadhyay et al. (2016). Later, they also observed that a similar phenomenon influences the migration characteristics of a drop in Poiseuille flow (Mandal et al., 2016b). The coupling of an external flow field and electrohydrodynamics can exhibit various non-intuitive flow physics in and around an individual drop (Allan \& Mason, 1962; Maehlmann \& Papageorgiou, 2009) and thus has the ability to drastically alter the bulk rheology of emulsions (Ha \& Yang, 2000; Fernndez, 2008). Vlahovska (2011) established a theoretical understanding of the impact of drop-level flow dynamics and deformation on the electrorheology of a dilute emulsion. They considered a uniform electric field in the direction of velocity gradient and analyzed the 
combined interplay among the flow and electric field in the limits of weak flow and highly viscous drops. The charge convection was neglected in the weak flow analysis. The orientation of the deformed drop along the flow direction was found responsible for the shear thinning effect due to reduction in the flow resistance. Similarly the emulsion shows shear thickening property when the drop elongates perpendicular to the direction as mentioned above. Apart from the shear rate dependent rheology, the electric field induced compression or elongation of drops causing an extra tension along the streamlines. As a result, the suspension possess normal stress differences, similar to polymeric liquids (Bird et al., 1987). Later Mandal \& Chakraborty (2017a) brought out the role of charge convection using an asymptotic analysis. Subsequently they showed (Mandal \& Chakraborty, 2017b) that application of an electric field in the shearing plane and along a tilted direction to that of the flow, can give rise to many non-intuitive bulk rheological properties of the emulsion, especially the existence of an electrical component of effective shear viscosity in the absence of drop deformation, which makes it deviate significantly from the result of (Taylor, 1932).

In many industrial applications the emulsion stabilizers act as a surface-active agents and reduce the interfacial tension after being adsorbed at the interface (Fischer \& Erni, 2007). Under dynamic conditions, these surface active agents or surfactants bring Marangoni stress in the picture and complicates the interfacial stress balance. Experiments in the literature have revealed the significant role of surfactants on the deformation of drops and the bulk rheology of an emulsion (Velankar et al., 2001, 2004; Jeon \& Macosko, 2003, Hu \& Lips, 2003). To analyze the specific physical phenomena responsible for such behavior, both analytical and numerical investigations have been performed (Stone \& Leal, 1990; Milliken et al., 1993; Li \& Pozrikidis, 1997; Vlahovska et al., 2005, 2009). These analyses show that the surfactant effects are mostly governed by the competing processes of surface convection, dilution and tip streaming. Depending on these mechanisms, the local surface tension on the drop varies up to a great extent, resulting in either suppression or enhancement of drop deformation. Several factors, such as the viscosity contrast of the drop-matrix fluid pair, local gradient in surface tension, initial surface tension and the flow strength, control the dominance among the said mechanisms.

The effect of surfactants on the electrically actuated drops, is a less studied domain in the literature (Ha \& Yang, 1995; Teigen \& Munkejord, 2010; Nganguia et al., 2013; Poddar et al. 2018; Ervik et al., 2018). The electrorheology of concentrated emulsions in the presence of nonionic surfactants has only been experimentally investigated by Ha et al. (1999). However, a theoretical understanding of the physical processes associated with drop scale flow physics and its connection to the bulk rheology of the effective fluid mixture, are missing in the literature. Thus in the present work we attempt to unveil the complicated interconnection between the surfactant covering on the drop surface, the electric field and the imposed linear shear flow, towards influencing the effective rheology. Considering non-ionic, bulk insoluble surfactant coating on the surface of a deformable viscous drop, dispersed in a electrically actuated emulsion, we adopt a comprehensive three-dimensional framework and analyze the problem through an asymptotic approach. Our results indicate that under the combined action of a tilted electric field and linear shear, the surfactant-induced Marangoni stresses modify the flow field around the drop in a diverse fashion, which cannot be predicted by a simple linear superposition of the governing processes. Consequently the bulk emulsion viscosity can either increase or decrease, 
depending on the choice of the ratio of dielectric permittivity and electrical conductivity combination of the two mediums. We also observed the unique existence of a critical shear rate relative to the electrical stresses, where the Marangoni effects vanish. Moreover, depending on the interplay between the charge convection and Marangoni effects, the emulsion rheology may become either shear thinning or shear thickening. Both the mechanisms of charge convection and small shape deformation of drops, have the ability to shift the critical shear rate observed in the leading order of case. Finally, depending on the tilt angle of the applied electric field, the elastic behavior of the emulsion, interpreted through normal stress differences, gets significantly altered due to the presence of interfacial tension gradients brought in by the surface surfactant distribution.

\section{Physical description}

In the present work, we consider a model emulsion where a drop of radius ' $a$ ' is dispersed in a background linear shear flow of the continuous phase along the $x$ direction given by $\widetilde{u}_{\infty}=\widetilde{G} \widetilde{y}$. Here $\widetilde{G}$ is the uniform shear rate on the external fluid flow in the far stream. The drop is being acted upon by a uniform magnitude DC electric field $\widetilde{\mathbf{E}}_{\infty}$ with a tilt angle $\Phi_{t}$ in the plane of shear. In addition, the drop surface is covered with non-ionic surfactant molecules. As a first step towards understanding the drop scale flow dynamics, we neglect the interaction between the drops and assume that the drop phase volume fraction $\nu$ to be in the dilute limit $(\nu \ll 1$, where $\nu$ is the drop phase concentration in the emulsion) and analyze the problem in a steady state condition. The scenario is shown schematically in figure 11(a). When the drop surface is uncontaminated the drop-matrix interface has a uniform surface tension $\widetilde{\gamma}_{c l}$. The presence of surface active agents on this interface lowers this surface tension and in the quiescent condition the surfactants has a uniform surface concentration of $\widetilde{\Gamma}_{e q}$. Under the simultaneous action of the background flow and the electric field the equilibrium distribution of the surfactants gets disrupted and the concentration distribution in the dynamic condition is given as $\widetilde{\Gamma}(\theta, \phi)$. Depending on the flow behavior and its surface properties, the drop surface may get deform to an ellipsoidal shape with its major axis being aligned with the $x$ direction at an angle $\varphi_{d}$. Two of the many possible configurations of the deformed drop in the $x-y$ plane are shown in figure 1(b). A body fitted coordinate system fixed at the drop centroid is also shown in the figure. The drop phase physical properties are denoted as: viscosity $\left(\mu_{d}\right)$, electrical conductivity $\left(\sigma_{d}\right)$ and dielectric permittivity $\left(\epsilon_{d}\right)$ while the corresponding quantities for the outer continuous phase are denoted with the subscript ' $d$ ' replaced with ' $c$ '. We assume that the drop is neutrally buoyant in the continuous fluid i.e. the densities of the two mediums are nearly equal $\left(\rho_{d} \approx \rho_{c}\right)$.

\section{Mathematical description}

In order to derive the dimensionless governing differential equations depicting the above physical problem we adopt the following characteristic values as reference scales for the different variables: (i) length $\sim a$, (ii) velocity $\sim \widetilde{G} a$, (iii) electric field $\sim \widetilde{E}_{\infty}$ and (iv) surfactant concentration $\sim \widetilde{\Gamma}_{e q}$. The relative importance of the characteristic electric stress $\left(\sim \epsilon_{c} \widetilde{E}_{\infty}^{2}\right)$ and hydrodynamic stress $\left(\sim \mu_{c} \widetilde{G}\right)$ is embodied in the dimensionless quantity defined as the Ma- 


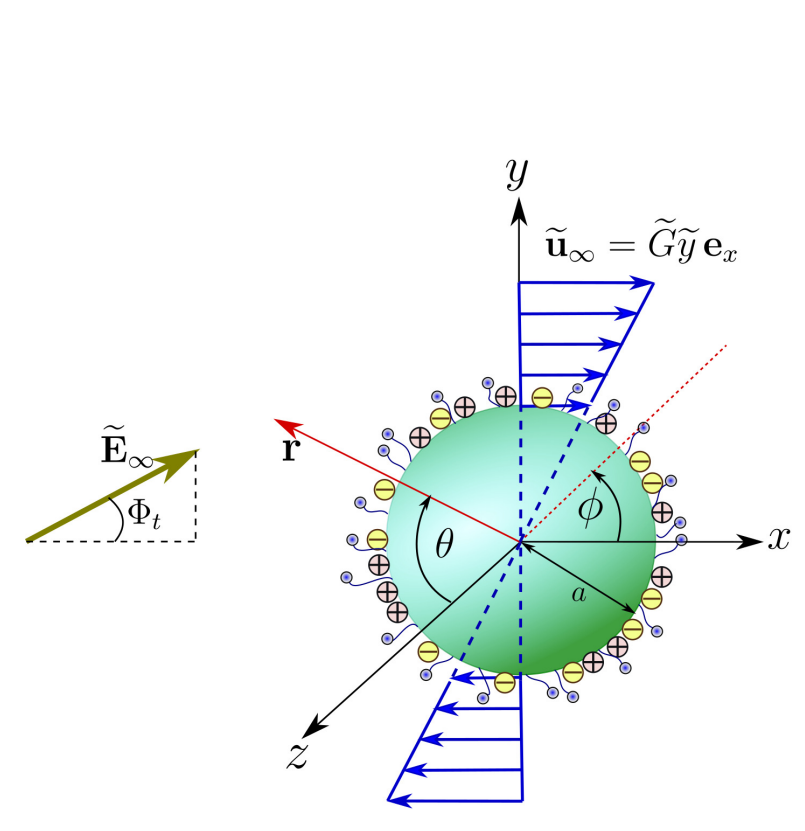

(a)
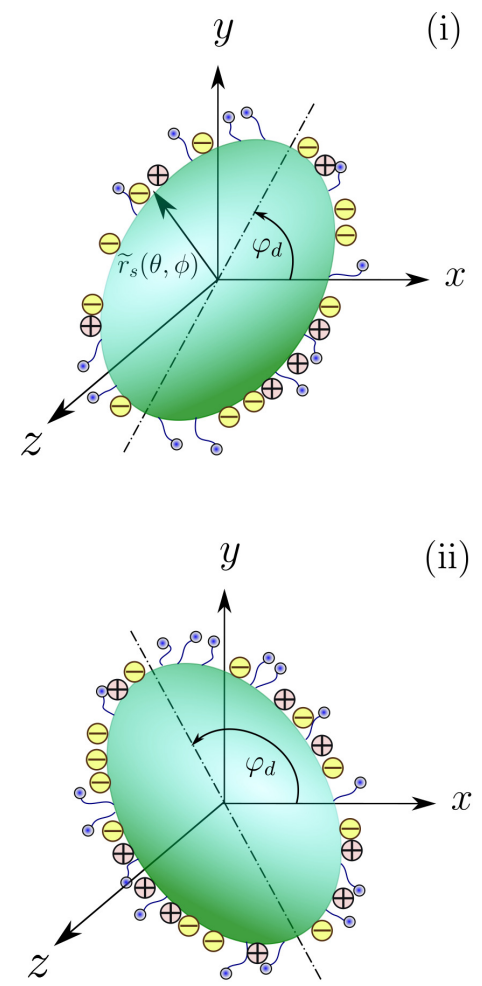

(b)

Figure 1: Schematic description of a deformable viscous drop being acted upon by a tilted electric field and background linear shear flow. The drop is covered with non-ionic surfactant molecules and interfacial charges accumulate on its surface. In sub-figure (a) the initial undeformed condition of the drop is shown. Under the action of the external fields the drop may deform with an inclination angle $\varphi_{d}$ as shown in sub-figure (b). Two of the many possible configurations with $\varphi_{d}<\pi / 2$ and $\varphi_{d}>\pi / 2$ are shown as configuration (i) and (ii), respectively.

son number, $M=\frac{\epsilon_{c} \widetilde{E}_{\infty}^{2}}{\mu_{c} \widetilde{G}}$. Due to this non-dimensionlization scheme we obtain the following important property ratios appearing in the problem formulation: electrical conductivity ratio, $R=\sigma_{d} / \sigma_{c}$, dielectric permittivity ratio, $S=\epsilon_{d} / \epsilon_{c}$ and viscosity ratio, $\lambda=\mu_{d} / \mu_{c}$.

\subsection{Governing equations and boundary conditions}

We assume that the concentration of the adsorbed surfactants on the drop interface is small so that it does not deviate significantly from the equilibrium concentration. Thus the surface tension, $\widetilde{\gamma}$ can be related to the local surfactant concentration by the relation (Leal, 2007; Stone, 1990)

$$
\widetilde{\gamma}(\widetilde{\Gamma})=\widetilde{\gamma}_{c l}-R_{g} T \widetilde{\Gamma},
$$

where $\widetilde{\gamma}_{c l}$ is the surface tension of a clean (surfactant-free) drop, $T$ stands for the absolute temperature and $R_{g}$ denotes the ideal gas constant. Further, $\widetilde{\gamma}_{c l}$ is related to the equilibrium interfacial tension as $\widetilde{\gamma}_{e q}=\widetilde{\gamma}_{c l}-R_{g} T \widetilde{\Gamma}_{e q}$. Hence equation (1) takes the alternative form

$$
\widetilde{\gamma}(\widetilde{\Gamma})=\widetilde{\gamma}_{e q}+R_{g} T\left(\widetilde{\Gamma}_{e q}-\widetilde{\Gamma}\right)
$$

Here onwards we denote the variables in their dimensionless forms and drop the " $\sim$, symbol from the notations. Now based on the present considerations of a steady state process and bulk 
insoluble surfactant molecules, the surfactant transport is governed by the following convectiondiffusion equation (Leal, 2007; Mandal et al., 2016a)

$$
P e_{S} \nabla_{S} \cdot\left(\mathbf{u}_{S} \Gamma\right)=\nabla_{S}^{2} \Gamma
$$

where $P e_{S}=a^{2} \widetilde{G} / D_{S}$ is the surface Péclet number which represents the relative importance of the convection and diffusion at the drop interface. In order to model the electrical effects we consider the situation when both the drop and continuous phases are leaky dielectric in nature and adopt the Taylor-Melcher leaky dielectric model Melcher \& Taylor, 1969, Saville, 1997; Vlahovska, 2019). According to this model free charge accumulation at the interface takes place due to finite Ohmic conductivities of the constituent fluidic phases. Now the time scale associated with the charge relaxation on account of electrical conduction from the bulk fluid to the drop-carrier fluid interface $\left(\tau_{C R}=\epsilon / \sigma\right)$ turns out to be significantly shorter than the time scale for convective process. As a consequence the volumetric charges may be neglected, allowing the momentum equation to be decoupled from electrostatics. However the finite electrical conductivities of fluid phases allow the free charge accumulation at the interface. This effectively reduces the volumetric free charge density to a boundary condition on the drop surface. Similarly, the ionic diffusion time scale turns out to be considerably higher than $\tau_{C R}$ leading to the assumption of zero bulk charge density. Thus the electric potential in the drop and continuous phase $\left(\psi_{d}, \psi_{c}\right)$ satisfy

$$
\nabla^{2} \psi_{d, c}=0
$$

Apart from the usual boundedness of potential in and around the drop, the matching condition of at the drop interface and the condition of uniformly imposed electric field at the far stream (i.e. $r \rightarrow \infty, \nabla \psi_{c}=-\mathbf{E}_{\infty}$ ), the interfacial charge balance turns out to an important condition for the present problem. In the quasi-steady state this reads (Xu \& Homsy, 2006)

$$
\text { at } r=r_{S}(\theta, \phi), \quad \mathbf{n} \cdot\left(R \nabla \psi_{d}-\nabla \psi_{c}\right)=-R e_{E} \nabla_{S} \cdot\left(q_{S} \mathbf{u}_{S}\right),
$$

where $\mathbf{n}$ is the unit vector in the normal direction at the drop surface and $\nabla_{S}$ is the surface gradient operator $\left(\nabla_{S}=(\mathbf{I}-\mathbf{n n}) \cdot \nabla\right)$. Here $R e_{E}=\epsilon_{c} \widetilde{G} / \sigma_{c}$ is the electrical Reynolds number quantifying the relative importance of the charge relaxation time scale and charge convection time scale. Also the charge density is calculated as

$$
q_{S}(\theta, \phi)=\left.\mathbf{n} \cdot\left(S \nabla \psi_{d}-\nabla \psi_{c}\right)\right|_{r=r_{S}} .
$$

The charge convection equation (5) suggests that the charge distribution and in effect the electric potential are coupled with the velocity field. Now since the velocity field is affected by the surfactant non-uniformity, due to the coupling as suggested by the equation (5), the electrical potential is also influenced by the surfactant.

Neglecting the inertial effect in the creeping flow limit $(R e \ll 1)$ the hydrodynamics can be described by the following equations (Happel \& Brenner, 1981)

$$
\begin{array}{ll}
-\nabla p_{d}+\lambda \nabla^{2} \mathbf{u}_{d}=0, & \nabla \cdot \mathbf{u}_{d}=0 \\
-\nabla p_{c}+\nabla^{2} \mathbf{u}_{c}=0, & \nabla \cdot \mathbf{u}_{c}=0
\end{array}
$$

Similar to the electric potential the pressure and velocity both in the drop as well as in the outer region must be bounded and they are continuous at the drop interface. Also the fluid 
velocity at the far stream matches with the applied background flow velocity i.e. as $r \rightarrow \infty$, $\mathbf{u}_{c}=\mathbf{V}_{\infty}$. Again in the quasi-steady state the normal velocity at the interface becomes zero i.e. at $r=r_{S}(\theta, \phi) \quad \mathbf{u}_{d} \cdot \mathbf{n}=\mathbf{u}_{c} \cdot \mathbf{n}=0$. Finally the interface stress balance can be expresses as:

$$
\text { at } r=r_{S}(\theta, \phi), \quad \llbracket \boldsymbol{T}^{H} \rrbracket+M \llbracket \boldsymbol{T}^{E} \rrbracket=\frac{1}{C a}(\nabla \cdot \mathbf{n}) \mathbf{n}+\underbrace{M a\left[(1-\Gamma)(\nabla \cdot \mathbf{n}) \mathbf{n}+\nabla_{S} \Gamma\right]}_{\text {Marangoni stress }},
$$

where $\llbracket \boldsymbol{\chi} \rrbracket$ denotes the jump of a variable $\boldsymbol{\chi}$ at the interface (e.g. $\left.\llbracket \chi \rrbracket=\chi_{c}-\chi_{d}\right) ; \boldsymbol{T}^{H, E}=\mathbf{n} \cdot \boldsymbol{\tau}^{H, E}$ are the viscous (superscript- $H$ ) and electrical (superscript- $E$ ) traction vectors at the interface. The Marangoni number $(M a)$ defined as $M a=R_{g} T \widetilde{\Gamma}_{e q} / \mu_{c} a \widetilde{G}$, as appeared in equation (8), represents the relative importance of the characteristic Marangoni stress due to surfactants and the characteristic viscous stress. Also $\mathrm{Ca}$ is the Capillary number denoting the ratio of the relative magnitudes of viscous and capillary stresses at the interface. It is a non-dimensional representation of the deformability of the drop surface. $M a$ can be related to $C a$ by the relation $M a=\frac{\beta}{C a(1-\beta)}$ where elasticity number, $\beta$ is defined in the form $\beta=-\frac{d\left(\widetilde{\gamma} / \widetilde{\gamma}_{c}\right)}{d\left(\widetilde{\Gamma} / \widetilde{\Gamma}_{e q}\right)}=\frac{R_{g} T \widetilde{\Gamma}_{e q}}{\widetilde{\gamma}_{c}}$. The parameter $\beta$ signifies the sensitivity surface tension on the concentration of surfactant molecules on the drop interface. For most of the practical purposes $\beta$ varies between 0 and 0.8 (Stone, 1990; Li \& Pozrikidis, 1997). The effects of Marangoni stress on tangential and normal stress balance at the drop interface can be visualized when the two components of the stress balance are considered separately as:

Tangential stress balance: at $r=r_{S}(\theta, \phi), \llbracket T_{t}^{H} \rrbracket+M \llbracket T_{t}^{E} \rrbracket=\underbrace{\frac{\beta}{C a(1-\beta)}\left(\nabla_{S} \Gamma\right) \cdot \mathbf{t}}_{\text {Marangoni effect }}$,

and

Normal stress balance: at $r=r_{S}(\theta, \phi), \quad \llbracket T_{n}^{H} \rrbracket+M \llbracket T_{n}^{E} \rrbracket=\frac{1}{C a}(\nabla \cdot \mathbf{n})+\underbrace{\frac{\beta}{C a(1-\beta)}[(1-\Gamma)(\nabla \cdot \mathbf{n})]}_{\text {Marangoni effect }}$.

Here $\mathbf{t}$ is the unit vector in the tangential direction at the drop surface.

\subsection{Asymptotic solution}

The governing equations depicting the present physical problem are coupled in such a manner that an exact solution for arbitrary values of the dimensionless parameters is not possible. To circumvent this problem we consider a physically realistic condition for which the charge convection effect is weak in comparison to the Ohmic conduction (i.e. $R e_{E} \ll 1$ ) and the drop deviates only slightly from the initial spherical shape (i.e. $C a \ll 1$ ) (Xu \& Homsy, 2006; Mandal et al. 2016b). In addition we consider that the surface Péclet number $\left(P e_{S}\right)$ is low (i.e. $\left.P e_{S} \ll 1\right)$ (Pak et al., 2014; Mandal et al., 2017b; Ha \& Yang, 1995), depicting the condition when the interfacial transport is mainly governed by the diffusion mechanism rather than convection. Thereafter a dimensionless physicochemical constant, $k$ can be defined as 
(Stone, 1990; Mandal et al., 2016a)

$$
k=\frac{P e_{S}}{C a}=\frac{\widetilde{\gamma}_{e q} a}{\mu_{c} D_{S}} .
$$

In this context, the physical condition of $k=0$ stands for a uniformly contaminated drop (Stone, 1990). Now for the assumed situation of $C a \ll 1, P e_{S} \ll 1$, the parameter $k$ turns out to be $\sim O(1)$. These considerations let us adopt a regular perturbation scheme which for any generic field variable $\chi$ reads

$$
\chi=\chi^{(0)}+C a \chi^{(C a)}+R e_{E} \chi^{\left(R e_{E}\right)}+O\left(C a^{2}, C a R e_{E}, R e_{E}^{2}\right) .
$$

Since the pressure and stress inside the drop must balance the capillary pressure in the static condition, they are to be expanded as (Chan \& Leal, 1979; Bandopadhyay et al., 2016):

$$
\left.\begin{array}{l}
p_{d}=\frac{1}{C a} p_{d}^{(1 / C a)}+p_{d}^{(0)}+C a p_{d}^{(C a)}+R e_{E} p_{d}^{\left(R e_{E}\right)}+O\left(C a^{2}, C a R e_{E}, R e_{E}^{2}\right) \\
\boldsymbol{\tau}_{\boldsymbol{d}}=\frac{1}{C a} \boldsymbol{\tau}_{\boldsymbol{d}}^{(1 / C a)}+\boldsymbol{\tau}_{\boldsymbol{d}}^{(0)}+C a \boldsymbol{\tau}_{\boldsymbol{d}}^{(C a)}+R e_{E} \boldsymbol{\tau}_{\boldsymbol{d}}{ }^{\left(R e_{E}\right)}+O\left(C a^{2}, C a R e_{E}, R e_{E}^{2}\right)
\end{array}\right\}
$$

Solving the normal stress equation equation 10 in different order of perturbation the drop shape is obtained in the form

$$
r_{S}=1+C a f^{(C a)}+C a R e_{E} f^{\left(C a R e_{E}\right)}+C a^{2} f^{\left(C a^{2}\right)}+\ldots
$$

In a similar fashion the interfacial surfactant concentration $\Gamma$ can be expressed as Stone, 1990; Ha \& Yang, 1995)

$$
\Gamma=\Gamma^{(0)}+C a \Gamma^{(C a)}+C a R e_{E} \Gamma^{\left(C a R e_{E}\right)}+C a^{2} \Gamma^{\left(C a^{2}\right)}+\ldots
$$

The local surfactant concentration also has to satisfy conservation of mass for the surfactant molecules which is mathematically denoted as

$$
\int_{\phi=0}^{2 \pi} \int_{\theta=0}^{\pi} \Gamma(\theta, \phi) r_{S}^{2}(\theta, \phi) \sin \theta d \theta d \phi=4 \pi
$$

Based on the above perturbation scheme the linearized hydrodynamic equations are solved using the generalized Lamb solution technique (Happel \& Brenner, 1981; Bandopadhyay et al. 2016). Accordingly the velocity and pressure field inside $\left(\mathbf{u}_{d}, p_{d}\right)$ and outside $\left(\mathbf{u}_{c}, p_{c}\right)$ the drop can be expressed a series of solid spherical harmonics given as (Haber \& Hetsroni, 1971, 1972)

$$
\begin{gathered}
\mathbf{u}_{d}=\sum_{n=1}^{\infty}\left[\nabla \times\left(\mathbf{r} \chi_{n}\right)+\nabla \Phi_{n}+\frac{n+3}{2(n+1)(2 n+3) \lambda} r^{2} \nabla p_{n}-\frac{n}{(n+1)(2 n+3) \lambda} \mathbf{r} p_{n}\right] \\
\text { and } p_{d}=\sum_{n=1}^{\infty} p_{n} \\
\mathbf{u}_{c}=\mathbf{V}_{\infty}+\sum_{n=1}^{\infty}\left[\nabla \times\left(\mathbf{r} \chi_{-n-1}\right)+\nabla \Phi_{-n-1}-\frac{n-2}{2 n(2 n-1)} r^{2} \nabla p_{-n-1}+\frac{n+1}{n(2 n-1)} \mathbf{r} p_{-n-1}\right]
\end{gathered}
$$




$$
\text { and } \quad p_{c}=\sum_{n=1}^{\infty} p_{-n-1}
$$

The different growing and decaying harmonics appearing in the above equations can be expressed as

$$
\begin{gathered}
p_{n}=\lambda r^{n} \sum_{m=0}^{n}\left[A_{n, m} \cos (m \phi)+\hat{A}_{n, m} \sin (m \phi)\right] P_{n, m}(\eta) \\
\left.\Phi_{n}=r^{n} \sum_{m=0}^{n}\left[B_{n, m} \cos (m \phi)+\hat{B}_{n, m} \sin (m \phi)\right] P_{n, m}(\eta)\right\} \\
\left.\chi_{n}=r^{n} \sum_{m=0}^{n}\left[C_{n, m} \cos (m \phi)+\hat{C}_{n, m} \sin (m \phi)\right] P_{n, m}(\eta)\right\} \\
p_{-n-1}=r^{-n-1} \sum_{m=0}^{n}\left[A_{-n-1, m} \cos (m \phi)+\hat{A}_{-n-1, m} \sin (m \phi)\right] P_{n, m}(\eta) \\
\Phi_{-n-1}=r^{-n-1} \sum_{m=0}^{n}\left[B_{-n-1, m} \cos (m \phi)+\hat{B}_{-n-1, m} \sin (m \phi)\right] P_{n, m}(\eta) \\
\chi_{-n-1}=r^{-n-1} \sum_{m=0}^{n}\left[C_{-n-1, m} \cos (m \phi)+\hat{C}_{-n-1, m} \sin (m \phi)\right] P_{n, m}(\eta)
\end{gathered},
$$

where $P_{n, m}(\eta)$ stands for the associated Legendre polynomial of degree $n$ and order $m$ with an argument $\eta=\cos (\theta)$. The various arbitrary constants $\left(A_{n}, B_{n}, C_{n}, A_{-n-1}, B_{-n-1}, C_{-n-1}\right.$, $\hat{A}_{n}, \hat{B}_{n}, \hat{C}_{n}, \hat{A}_{-n-1}, \hat{B}_{-n-1}$ and $\left.\hat{C}_{-n-1}\right)$ are to be determined by simultaneously solving the surfactant transport equation and the appropriate form of the hydrodynamic boundary conditions. The detailed procedure regarding the treatment of these boundary conditions is tedious and can be found in earlier studies (Haber \& Hetsroni, 1972; Bandopadhyay et al., 2016).

The governing equations electrical potential (equation (40) in both the drop and continuous phase are solved by expanding the potential in the form

$$
\left.\begin{array}{c}
\psi_{d}=\sum_{n=0}^{\infty} r^{n} \sum_{m=0}^{n}\left[a_{n, m} \cos (m \phi)+\hat{a}_{n, m} \sin (m \phi)\right] P_{n, m}(\cos (\theta)) \\
\psi_{\infty}+\sum_{n=0}^{\infty} r^{-n-1} \sum_{m=0}^{n}\left[b_{-n-1, m} \cos (m \phi)+\hat{b}_{-n-1, m} \sin (m \phi)\right] P_{n, m}(\cos (\theta))
\end{array}\right\},
$$

where $\psi_{\infty}$ takes care of the far field condition of a uniform tilted electric field.

We seek solutions for the shape function $f(\theta, \phi)$ and the surfactant concentration, $\Gamma(\theta, \phi)$ by expanding them in terms of surface harmonics as:

$$
f=\sum_{n=0}^{\infty} \sum_{m=0}^{n}\left[L_{n, m} \cos (m \phi)+\hat{L}_{n, m} \sin (m \phi)\right] P_{n, m}(\eta)
$$

and

$$
\Gamma=\sum_{n=0}^{\infty} \sum_{m=0}^{n}\left[\Gamma_{n, m} \cos (m \phi)+\hat{\Gamma}_{n, m} \sin (m \phi)\right] P_{n, m}(\eta)
$$




\subsection{Emulsion rheology}

In the present physical situation, the simultaneous action of the imposed shear flow and a uniform electric field significantly alters the flow field around the drop and also modifies the drop shape. The presence of surfactant induced Marangoni stress further complicates the scenario. Thus the effective rheology of the emulsion containing the drops is expected to deviate from the condition when the surrounding fluid is considered alone. The contribution of micro level flow physics in the dispersed phase to the effective macro-scale rheology of a dilute emulsion can be calculated by determining a volume averaged stress of the emulsion (Schowalter et al. 1968; Batchelor, 1970; Guazzelli \& Morris, 2011): .

$$
\langle\boldsymbol{\tau}\rangle=-\langle p\rangle \mathbf{I}+2 \mathbf{D}_{\infty}+\frac{\nu}{V_{d}} \mathbf{S}
$$

Here $\mathbf{D}_{\infty}=\left(\nabla \mathbf{V}_{\infty}+\left(\nabla \mathbf{V}_{\infty}\right)^{T}\right) / 2$ is the straining component of the externally imposed linear shear flow and $\mathbf{S}$ is the stresslet defined as

$\mathbf{S}=\int_{\phi=0}^{2 \pi} \int_{\theta=0}^{\pi}\left[\frac{1}{2}\left(\left(\boldsymbol{\tau}_{c}^{H} \cdot \mathbf{n}\right) \mathbf{r}+\left(\left(\boldsymbol{\tau}_{c}^{H} \cdot \mathbf{n}\right) \mathbf{r}\right)^{T}-\frac{2}{3} \mathbf{I}\left(\boldsymbol{\tau}_{c}^{H} \cdot \mathbf{n}\right) \mathbf{r}\right)-\left(\mathbf{u}_{\mathbf{c}} \mathbf{n}+\left(\mathbf{u}_{\mathbf{c}} \mathbf{n}\right)^{T}\right)\right] r_{S}^{2} \sin (\theta) d \theta d \phi$.

The stresslet represents the change in the total stress brought in by the velocity and stress modifications in the continuous phase by the presence of the drop phase. Following the presently adopted Lamb solution technique mentioned in section 3.2 , the stresslet can be alternatively expressed in a more compact form as a function of the decaying solid spherical harmonic $p_{3}$ as: (Happel \& Brenner, 1981; Kim \& Karila, 1991)

$$
\mathbf{S}=-\frac{\nu}{2} \nabla \nabla\left(r^{5} p_{-3}\right)
$$

The heterogeneity in the emulsion is quantified by defining an effective shear viscosity of emulsion, the dimensionless form of which is given as (Schowalter et al., 1968; Bird et al., 1987)

$$
\eta_{\mathrm{eff}}=\frac{\mu_{\mathrm{eff}}}{\mu_{c}}=\langle\boldsymbol{\tau}\rangle_{x y}
$$

The isotropy of normal stress is disturbed in a sheared emulsion (Guazzelli \& Morris, 2011). This anisotropy is quantified by the first and second normal stress differences which are calculated as:

$$
\text { first normal stress difference: } \quad N_{1}=\frac{\langle\widetilde{\boldsymbol{\tau}}\rangle_{x x}-\langle\widetilde{\boldsymbol{\tau}}\rangle_{y y}}{\mu_{c} \widetilde{G}}=\langle\boldsymbol{\tau}\rangle_{x x}-\langle\boldsymbol{\tau}\rangle_{y y}
$$

and

$$
\text { second normal stress difference: } \quad N_{2}=\frac{\langle\widetilde{\boldsymbol{\tau}}\rangle_{y y}-\langle\widetilde{\boldsymbol{\tau}}\rangle_{z z}}{\mu_{c} \widetilde{G}}=\langle\boldsymbol{\tau}\rangle_{y y}-\langle\boldsymbol{\tau}\rangle_{z z} \text {. }
$$

We proceed to calculate the electric potential, flow field and the surfactant distribution in the different orders of perturbation, namely the leading order $O(1)$, the $O\left(R e_{E}\right)$ and the $O(C a)$. Next the effective emulsion stress and the normal stress difference expressions of orders $O(\nu), O\left(\nu R e_{E}\right)$ and $O(\nu C a)$ are determined by considering a dilute emulsion $(\nu \ll 1)$ where drop-drop interactions are not significant. The detailed solution strategy at different perturbation orders are given in earlier studies (Bandopadhyay et al., 2016; Mandal et al., 2016b) and hence in the following sections we only provide the expressions of different physical variables which are important for the present study. 


\subsection{Description at leading order}

The leading order description of different variables are obtained by considering an undeformed drop for which the charge convection at the surface is too small to be taken into account. Thus in this order of perturbation the electrical potential distribution becomes independent of any flow induced effect and can be solely expressed in terms of electrical property ratios as given below:

$$
\begin{gathered}
\psi_{d}^{(0)}=-\frac{3 r}{R+2} \cos \left(\Phi_{t}-\phi\right) P_{1,1}(\eta), \\
\psi_{c}^{(0)}=\left(\frac{1}{r^{2}} \frac{R-1}{R+2}-r\right) \cos \left(\Phi_{t}-\phi\right) P_{1,1}(\eta) .
\end{gathered}
$$

Similarly the leading order interfacial charge density becomes

$$
q_{S}^{(0)}=\frac{3(R-S)}{R+2} \cos \left(\Phi_{t}-\phi\right) P_{1,1}(\eta) .
$$

The surfactant distribution up to $O(C a)$ can be expressed as

$$
\Gamma=\Gamma^{(0)}+\Gamma^{(C a)}=\Gamma^{(0)}+\Gamma_{2,0}^{(C a)} P_{2,0}(\eta)+\Gamma_{2,2}^{(C a)} \cos (2 \phi) P_{2,2}(\eta)+\hat{\Gamma}_{2,2}^{(C a)} \sin (2 \phi) P_{2,2}(\eta),
$$

where different harmonics of surfactant concentration are obtained as

$$
\begin{aligned}
& \Gamma^{(0)}=1 ; \\
& \Gamma_{2,0}^{(C a)}=-\frac{3}{2} \frac{M(1-\beta)(R-S) k}{(R+2)^{2}((k-5 \lambda-5) \beta+5 \lambda+5)} ; \\
& \Gamma_{2,2}^{(C a)}=\frac{3}{4} \frac{M \cos \left(2 \Phi_{t}\right)(1-\beta)(R-S) k}{(R+2)^{2}((k-5 \lambda-5) \beta+5 \lambda+5)} ; \\
& \hat{\Gamma}_{2,2}^{(C a)}=\frac{5}{12} \frac{(1-\beta) k}{(k-5 \lambda-5) \beta+5 \lambda+5}+\frac{3}{4} \frac{M \sin \left(2 \Phi_{t}\right)(1-\beta)(R-S) k}{(R+2)^{2}((k-5 \lambda-5) \beta+5 \lambda+5)} .
\end{aligned}
$$

The leading order velocity and pressure fields, both inside the drop $\left(\mathbf{u}_{d}^{(0)}, p_{d}^{(0)}\right)$ and in the matrix phase $\left(\mathbf{u}_{c}^{(0)}, p_{c}^{(0)}\right)$, can be fully expressed in terms of the non-zero solid harmonics up to $n=2$ and all the other term vanish. Of particular interest is the leading order interfacial fluid velocity which can be expressed in the spherical coordinate system as $\mathbf{u}_{S}^{(0)}=u_{S, \theta}^{(0)} \mathbf{i}_{\theta}+u_{S, \phi}^{(0)} \mathbf{i}_{\phi}$, where the scalar components are given in the equation $\mathrm{A}-1$.

The leading order effective viscosity becomes a function of the arbitrary constant $\hat{A}_{-3,2}^{(0)}$ which is present in the decaying harmonics in equation (22) and is given by (Mandal \& Chakraborty, 2017b)

$$
\eta_{\mathrm{eff}}^{(0)}=1-3 \nu \hat{A}_{-3,2}^{(0)} .
$$

Finally $\eta_{\mathrm{eff}}^{(0)}$ can be written in the form

$$
\begin{aligned}
\eta_{\mathrm{eff}}^{(0)}= & \underbrace{1+\nu \frac{(5 \lambda+2)}{(2 \lambda+2)}}_{\eta_{\mathrm{h}, \text { clean }}^{(0)}}+\underbrace{\frac{3}{2} \frac{\nu k \beta}{((k-5 \lambda-5) \beta+5 \lambda}(1+\lambda)}_{\text {surfactant contribution }\left(\eta_{\mathrm{h}, \text { surf }}^{(0)}\right)} \\
& \underbrace{-\frac{27 M \nu \sin \left(2 \Phi_{t}\right)(R-S)}{10(\lambda+1)(R+2)^{2}}}_{\eta_{\mathrm{e}, \text { clean }}^{(0)}} \times \underbrace{\frac{(1-\beta)(1+\lambda)}{(k-5 \lambda-5) \beta+5 \lambda+5}}_{\text {surfactant contribution }\left(\eta_{\mathrm{e}, \text { surf }}^{(0)}\right)} .
\end{aligned}
$$


It is noteworthy to observe that surfactant induced Marangoni stress affects both the hydrodynamic $\left(\eta_{\mathrm{h}, \text { surf }}^{(0)}\right)$ and electrical $\left(\eta_{\mathrm{e}, \text { surf }}^{(0)}\right)$ contributions to the leading order effective viscosity. Moreover, the effect on the electrical component is of a complex functional form which is in contrast to the linear superposition similar to the hydrodynamic contribution as shown above.

In a similar manner the first and second normal stress differences are obtained as

$$
N_{1}^{(0)}=-6 \nu A_{-3,2}^{(0)}=-\frac{27 \nu M \cos \left(2 \Phi_{t}\right)(R-S)}{(\lambda+1)(R+2)^{2}} \times \underbrace{\frac{(1-\beta)(1+\lambda)}{(k-5 \lambda-5) \beta+5(\lambda+1)}}_{\mathcal{N}_{1, \text { sur }}^{(0)}=\text { surfactant contribution }}
$$

and

$$
N_{2}^{(0)}=\nu\left(3 A_{-3,2}^{(0)}+\frac{3}{2} A_{-3,0}^{(0)}\right)=-\frac{27 \nu M \sin ^{2}\left(\Phi_{t}\right)(R-S)}{(\lambda+1)(R+2)^{2}} \times \underbrace{\frac{(1-\beta)(1+\lambda)}{(k-5 \lambda-5) \beta+5(\lambda+1)}}_{\mathcal{N}_{2, \text { surf }}^{(0)}=\text { surfactant contribution }},
$$

where both the expressions contain a common surfactant contribution term,

$$
\mathcal{N}_{1, \text { surf }}^{(0)}=\mathcal{N}_{2, \text { surf }}^{(0)}=\mathcal{N}_{\text {surf }}^{(0)}=\frac{(1-\beta)(1+\lambda)}{(k-5 \lambda-5) \beta+5(\lambda+1)} .
$$

\subsection{Order $R e_{E}$ description}

In this case we consider an undeformed drop but take into account of the charge convection at the drop surface. In contrast to the leading order, the interfacial charge distribution of $O\left(R e_{E}\right)$ gets affected by the flow field and the surfactant effects and subsequently we get $q_{S}^{R e_{E}}=q_{S}^{R e_{E}}\left(\theta, \phi ; R, S, \lambda, M, \Phi_{t}, \beta, k\right)$ (see the supplementary material for the full expression of $\left.q_{S}^{\left(R e_{E}\right)}\right)$. This behavior can be understood by looking into the charge convection equation applicable for $O\left(R e_{E}\right)$ given as

$$
\text { at } r=1 \quad R \frac{\partial \psi_{d}}{\partial r}-\frac{\partial \psi_{c}}{\partial r}=-\nabla_{S} \cdot\left(q_{S}^{(0)} \mathbf{u}_{S}^{(0)}\right)
$$

The surfactant transport equation in this order of perturbation takes the form

$$
\text { at } r=1, \quad k \nabla_{S} \cdot\left(\mathbf{u}_{S}^{\left(R e_{E}\right)}\right)=\nabla_{S}^{2} \Gamma^{\left(C a R e_{E}\right)},
$$

where the surfactant concentration distribution is obtained as

$$
\Gamma^{\left(C a R e_{E}\right)}=\sum_{n=2}^{4} \sum_{m=0}^{n}\left(\Gamma_{n, m}^{\left(C a R e_{E}\right)} \cos (m \phi)+\hat{\Gamma}_{n, m}^{\left(C a R e_{E}\right)} \sin (m \phi)\right) P_{n, m}(\eta) .
$$

Again the harmonic constants $\left(\Gamma_{n, m}^{\left(C a R e_{E}\right)}, \hat{\Gamma}_{n, m}^{\left(C a R e_{E}\right)}\right)$ can be found in the Supplementary Material. Here the non-zero solid harmonics upto $n=4$ are required to fully describe the pressure and velocity fields $\left(\mathbf{u}_{d}^{\left(R e_{E}\right)}, p_{d}^{\left(R e_{E}\right)}, \mathbf{u}_{c}^{\left(R e_{E}\right)}, p_{c}^{\left(R e_{E}\right)}\right)$. The full expressions are not mentioned due to their excessive lengths but can be found in the supplementary material

The effective viscosity is obtained as a correction to the leading order term, i.e. $\eta_{\text {eff }}=$ $\eta_{\mathrm{eff}}^{(0)}+R e_{E} \eta_{\mathrm{eff}}^{\left(R e_{E}\right)}$. In terms of the arbitrary constants present in the decaying velocity field the $\eta_{\text {eff }}^{\left(R e_{E}\right)}$ can be written as (Mandal \& Chakraborty, 2017b)

$$
\eta_{\mathrm{eff}}^{\left(R e_{E}\right)}=-3 \nu \hat{A}_{-3,2}^{\left(R e_{E}\right)} .
$$


Unlike the leading order, the surfactant contribution parameters of order $R e_{E}$ are not equal, i.e. $\left(\mathcal{N}_{1}^{\left(R e_{E}\right)} \neq \mathcal{N}_{2}^{\left(R e_{E}\right)}\right)$. In addition to being functions of the surfactant parameters $\beta$ and $k$, they are also strongly dependent on electrical parameters, namely the tilt angle $\left(\Phi_{t}\right)$, the Mason number $(M)$ and the electrical property ratios $(R, S)$. This again suggests a coupled nature of the Marangoni effects on the electrical Maxwell stress.

\subsection{Order Ca description}

In this section we consider the deformable nature of the drop and disregard the charge convection effect. This is mathematically represented with the help of deformed drop radius, $r_{S}(\theta, \phi)=1+C a f^{(C a)}$. Now the value of any variable on the deformed surface can be expressed as a correction to the leading order solution, calculated using the Taylor series expansion as

$$
\left[\left.\chi\right|_{r=r_{S}}\right]^{(C a)}=\left.\chi^{(C a)}\right|_{r=1}+\left.f^{(C a)} \frac{\partial \chi^{(0)}}{\partial r}\right|_{r=1}
$$

where the shape function $f^{(C a)}$ is obtained by solving the normal stress balance at the leading order:

$$
\text { at } r=1, \quad \llbracket T_{r}^{H(0)} \rrbracket+M \llbracket T_{r}^{E(0)} \rrbracket=-\left(2 f^{(C a)}+\nabla^{2} f^{(C a)}\right)-\frac{2 \beta}{1-\beta} \Gamma^{(C a)} .
$$

The shape function has a form

$$
f^{(C a)}=\sum_{m=0}^{2}\left(L_{2, m}^{(C a)} \cos (m \phi)+\hat{L}_{2, m}^{(C a)} \sin (m \phi)\right) P_{2, m}(\eta),
$$

where the different non-zero deformation modes are obtained as

$$
\begin{aligned}
L_{2,0}^{(C a)}=\left.L_{2,0}^{(C a)}\right|_{\text {Clean }}-\frac{3}{40} & \frac{M k \beta(\lambda+4)(R-S)}{((-5 \beta+5) \lambda+5+(k-5) \beta)(R+2)^{2}(\lambda+1)} ; \\
L_{2,2}^{(C a)}=\left.L_{2,2}^{(C a)}\right|_{\text {Clean }}+\frac{3}{80} & \frac{M k \beta(\lambda+4)(R-S) \cos \left(2 \Phi_{t}\right)}{(\lambda+1)((-5 \beta+5) \lambda+5+(k-5) \beta)(R+2)^{2}} ; \\
\text { and } \hat{L}_{2,2}^{(C a)}=\left.\hat{L}_{2,2}^{(C a)}\right|_{\text {Clean }} & +\frac{1}{48} \frac{k \beta(\lambda+4)}{(\lambda+1)((-5 \beta+5) \lambda+5+(k-5) \beta)} \\
& +\frac{3}{80} \frac{k \beta(\lambda+4) M \sin \left(2 \Phi_{t}\right)(R-S)}{(\lambda+1)((-5 \beta+5) \lambda+5+(k-5) \beta)(R+2)^{2}} .
\end{aligned}
$$

In each of the above expressions the second term corresponds to the modification in the drop shape brought in by the non-uniform surfactant distribution. Interestingly, for the deformation modes $L_{2,0}^{(\mathrm{Ca})}, L_{2,2}^{(\mathrm{Ca})}$ the surfactant only has its role only if there is an applied electric field. On the other hand the form of $\hat{L}_{2,2}^{(\mathrm{Ca})}$ suggests that the deformation arising from the hydrodynamic origin is itself influenced by the Marangoni effect.

The surfactant transport equation takes a slightly different form than the previously discussed perturbation orders and is given by

$$
\text { at } r=1+C a f^{(C a)}, \quad k \nabla_{S} \cdot\left(\mathbf{u}_{S}^{(0)} \Gamma^{(C a)}+\mathbf{u}_{S}^{(C a)}\right)=\nabla_{S}^{2} \Gamma^{\left(C a^{2}\right)} .
$$

Determination of the complete $\mathrm{O}(\mathrm{Ca})$ flow field and surfactant distribution becomes too cumbersome to perform. However the main objective of the present work being the determination 
of the bulk rheology characteristics of the emulsion, it is required to calculate only the arbitrary constants $\hat{A}_{-3,2}^{(\mathrm{Ca})}, A_{-3,2}^{(\mathrm{Ca})}$ and $A_{-3,0}^{(\mathrm{Ca})}$ which are present in the $O(\mathrm{Ca})$ flow field. Using these constants we can express the $O(\mathrm{Ca})$ effective viscosity and normal stress differences as (Mandal \& Chakraborty, 2017b):

$$
\begin{aligned}
& \eta_{\mathrm{eff}}^{(C a)}=-3 \nu \hat{A}_{-3,2}^{(C a)} . \\
& N_{1}^{(C a)}=-6 \nu A_{-3,2}^{(C a)}
\end{aligned}
$$

and

$$
N_{2}^{(C a)}=\nu\left(3 A_{-3,2}^{(\mathrm{Ca})}+\frac{3}{2} A_{-3,0}^{(\mathrm{Ca})}\right) .
$$

The final forms of the $O(C a)$ bulk rheological parameters (please refer to the Supplementary Material for full expressions) again suggest a strong coupling between the Marangoni stress, hydrodynamic and electrical Maxwell stresses under the condition of a deformed drop surface.

\section{Results and Discussions}

In the above formulation of the coupled governing equations and boundary conditions, we have finally represented various physical variables in terms of a set of dimensionless parameters, given as $C a, M, \operatorname{Re}_{E}, R, S, \lambda, \Phi_{t}, \beta$ and $k$. To ensure the correctness of the present mathematical analysis, we consider different limiting conditions of the present study and compare the key results with those of the existing works in the literature (see section Appendix B for details). In the following discussions we will first analyze the effects of various electrohydrodynamic parameters on the interfacial distribution of the surfactant molecules. Next we discuss the impact of the coupled interplay of the surfactant parameters $\beta, k$ and electrical effects (characterized by the parameters $R, S, \Phi_{t}$ ) on the bulk emulsion rheology when the drop is undeformed and not affected by the charge convection. In the consequent subsections we delve deeper to investigate the individual effects of charge convection and small shape deformation on the emulsion rheology. The theoretical model presented above considers a parametric range of as $C a \ll 1, R e_{E} \ll 1, M \sim O(1)$. In addition we assume that the surface diffusion is the dominant transport mechanism for the surface active agents, $P e_{S} \ll 1$ (or $k \sim O(1)$ ).

Here we demonstrate the results by considering that both the dispersed and continuous phases are leaky dielectric in nature. To find the practical relevance of these assumptions we consider a model emulsion where a silicone oil drops $\left(\mu_{d}=1.29\right.$ Pas, $\sigma_{d}=5.8 \times$ $\left.10^{-12} \mathrm{~S} / \mathrm{m}, \epsilon_{d}=2.7 \epsilon_{0}\right)$ is dispersed in the continuous phase of castor oil $\left(\mu_{c}=1.29\right.$ Pas, $\sigma_{c}=$ $5.8 \times 10^{-10} \mathrm{~S} / \mathrm{m}, \epsilon_{c}=3.8 \epsilon_{0}$ ) (Ha et al., 1999$)$. Also the drop surface is covered with nonionic surfactants such as PL64 or Tween 60. The interfacial tension of uncontaminated drop is taken as $\widetilde{\gamma}_{c l}=4.6 \mathrm{mN} / \mathrm{m}$ ( $\mathrm{Ha} \&$ Yang, 2000). Considering $\beta=0.8$ and using the relation $\widetilde{\gamma}_{e q}=(1-\beta) \widetilde{\gamma}_{c l}$ we get the equilibrium surface tension for a surfactant coated drop in this case as $\widetilde{\gamma}_{e q}=0.92 \mathrm{mN} / \mathrm{m}$. Now if we choose a typical shear rate $\widetilde{G}=10 \mathrm{~s}^{-1}$, drop diameter of $30 \mu \mathrm{m}$ (e.g. in the experiments of Ha et al. (1999)), characteristic electric field strength of $\widetilde{E}_{\infty}=0.8 \mathrm{kV} / \mathrm{mm}$ and a surface diffusivity value of $D_{S}=10^{-8} \mathrm{~m}^{2} / \mathrm{s}$ Valkovska \& Danov, 2000) then the various dimensionless parameters relevant to the present problem can be calculated as $M=1.35, C a=0.26, R e_{E}=0.29$ and $P e_{S}=0.23$. Hence the perturbation scheme 
presented in section 3.2 can predict a practical experimental condition. The set of electrohydrodynamic property ratios $(R=0.005, S=0.71, \lambda=0.81$ ), discussed so far, is named as system- $A$ in the demonstrative examples to follow. Along with this, to bring out the contrast in results which the different combination of drop-matrix properties can exhibit, we have chosen two other combinations of the electrohydrodynamic property ratios given as system- $B: R=2.5, S=0.1$, $\lambda=0.1$ (Mandal et al. $2017 a)$; and system- $C: R=0.01, S=1.7, \lambda=0.03$ (Xu \& Homsy, $2006)$.

\subsection{Nonuniform surfactant distribution}

(a)

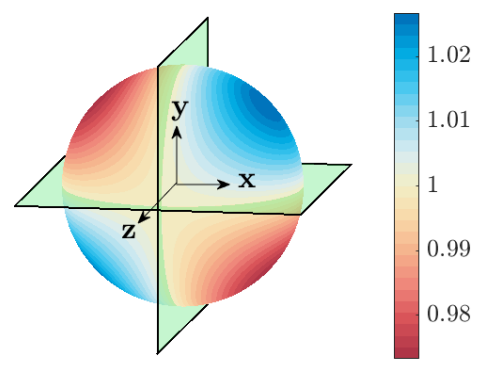

(b)
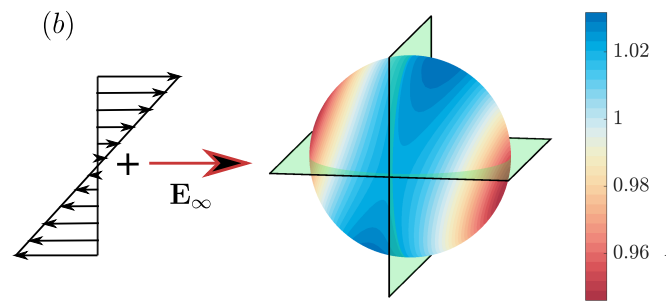

(c)
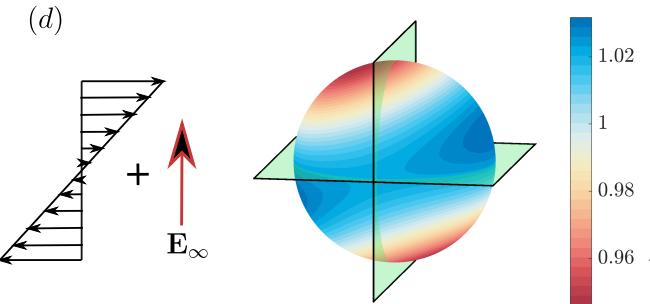

$(e)$

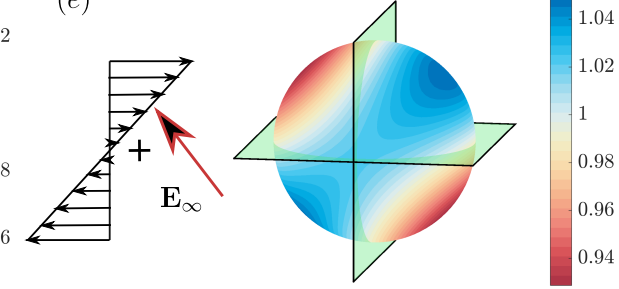

Figure 2: Effect of electric field on the surfactant concentration distribution on the drop surface $\Gamma=\Gamma^{(0)}+C a \Gamma^{(C a)}$. In subplot (a) only the effect of background shear flow is considered. In the subplots (b)-(e) the electrical effects are shown by varying the tilt angle of the applied electric field as $\Phi_{t}=0, \pi / 4, \pi / 2$ and $3 \pi / 4$, respectively. Here the other electrohydrodynamic parameters are chosen as per system-A. The other parameters are: Capillary number, $C a=0.2$; Mason number, $M=5$; the elasticity parameter, $\beta=0.8$ and physicochemical constant, $k=1$.

In the absence of any electric field, when only a linear shear flow is imposed, the surfactant molecules obtains a non-uniform but anti-symmetric distribution $(\Gamma(\theta, \phi))$ as shown in figure 2(a). The $\Gamma(\theta, \phi)$ follows the straining axis of the flow which is inclined at an angle $\phi=\pi / 4$ with the background flow direction (Bentley \& Leal, 1986). In that case, the surfactant molecules are observed to accumulate more towards the polar regions of this axis and a dominant depletion takes place orthogonal to these locations. But this anti-symmetric pattern 
changes when an electric field is applied (shown in figures 2 (b)-(e)). With the application of an external field the surfactant distribution does not follow the axisymmetric deposition along $\phi=\pi / 4$. While for the tilt angles $\Phi_{t}=0, \pi / 2$ and $3 \pi / 4$ this common trend is followed, a completely different characteristic of surfactant distribution emerges for $\Phi_{t}=\pi / 4$. In this case the maximum surfactant concentration points are no longer in the plane of shear $(x-y$ plane in this case) and the maxima points shift to $y-z$ plane which is orthogonal to the plane of shear. Such undulations in the local surfactant concentration trigger corresponding changes in the interfacial tension $\gamma(\theta, \phi)$ and gradients in surface tension $\left(\left|\gamma_{\max }-\gamma_{\min }\right|\right)$ along the drop surface. Along with the tilt angle of the applied electric field, the Mason number $(M)$, indicating the relative importance of the electrical stress in comparison to the hydrodynamic stress, also deeply influences the surface tension distribution along the interface. This influence is depicted in figure A-1 for the configuration of vertically applied electric field. It is observed that the symmetric deviation of the maximum and minimum surface tension about the equilibrium condition in the case of simple shear flow, is not maintained when electric field is applied. Also the gradients in surface tension $\left(\left|\gamma_{\max }-\gamma_{\min }\right|\right)$ become progressively higher as the influence of electrical stresses increase relative to their hydrodynamic counterparts.

The above characteristics of $\Gamma(\theta, \phi)$ under different conditions become clear by looking into the equation (35). It shows that different modes in $\Gamma^{(\mathrm{Ca})}$ contain the information of various aspects governing the flow physics. While $\Gamma_{2,0}^{(\mathrm{Ca})}$ and $\Gamma_{2,2}^{(\mathrm{Ca})}$ are only present when an external electric field is applied, the harmonic $\hat{\Gamma}_{2,2}^{(\mathrm{Ca})}$ has a non-zero component arising solely from hydrodynamic origin. Again, the surfactant harmonics are strongly dependent on the tilt angle of the applied electric field. $\Gamma_{2,0}^{(\mathrm{Ca})}$ appears for any configuration of the electric field, but $\Gamma_{2,2}^{(\mathrm{Ca})}$ vanishes completely when the tilt angle becomes $\Phi_{t}=\pi / 4$ or $3 \pi / 4$. Similarly the electric component of $\hat{\Gamma}_{2,2}^{(C a)}$ becomes zero when the electric field is applied in either horizontal $\left(\Phi_{t}=0\right)$ or vertical $\left(\Phi_{t}=\pi / 2\right)$ configuration. In order to investigate the physics behind the coupled behavior of imposed linear shear and electric field we appeal to the flow characteristics around the drop originating from the individual effects (Mandal \& Chakraborty, 2017b, Vlahovska, 2011). The linear shear flow can be decomposed as a combination of a pure straining and pure rotational flow. On the other hand, the externally applied tilted electric field can give rise to either a uniaxial (for $R>S$ ) or biaxial straining (for $R<S$ ) flow around the drop. Hence, under the simultaneous action of shear flow and electrically induced flow, the surfactant molecules assume diverse redistribution patterns, the final form of which is determined by a combined interfacial convection and diffusive transport.

\subsection{Leading order bulk rheology}

In figures 3 (a) to (c) the contribution of non-uniform surfactant distribution on the drop surface, in modulating the leading order normalized effective shear viscosity $\left(\bar{\eta}_{\mathrm{eff}}^{(0)}=\left(\eta_{\mathrm{eff}}^{(0)}-1\right) / \nu\right)$, is described. It is observed from figures 3 (a) and (b) that increase in either of the parameters $\beta$ and $k$ causes an enhancement in the effective viscosity. In contrast, figure 3 (c) depicts that the surfactant characterization parameters have a completely reverse effect on $\bar{\eta}_{\text {eff }}$. To understand the effects of the surfactant characterization parameters $\beta$ and $k$, we appeal to figure A-2 where the variations in surfactant concentration and surface tension are shown. The surface elasticity number $\beta$ signifies the sensitivity of the surface tension on the local surfactant concentration. 


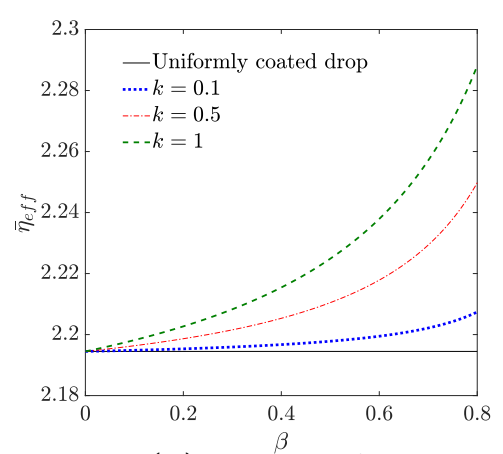

(a) System-A

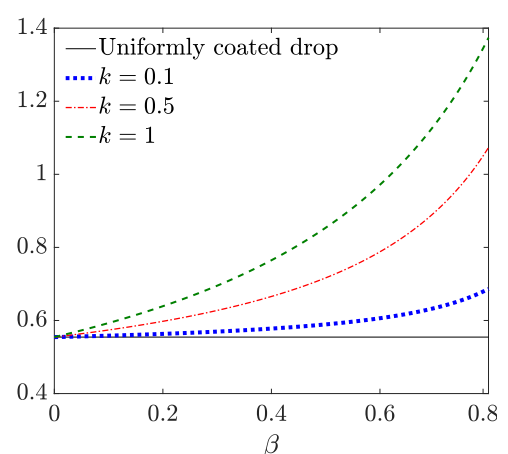

(b) System-B

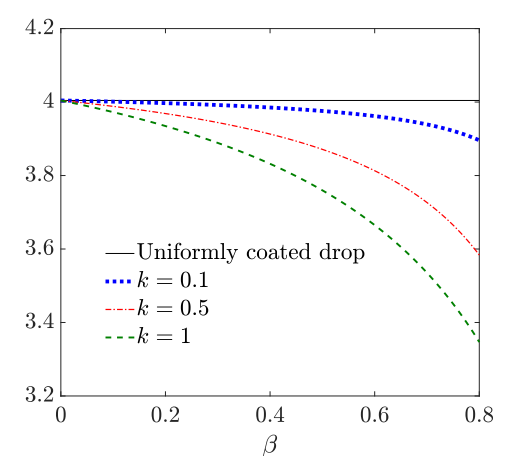

(c) System-C

Figure 3: Leading order normalized effective shear viscosity, $\bar{\eta}_{\text {eff }}=\left(\eta_{\text {eff }}^{(0)}-1\right) / \nu$ vs. elasticity parameter for different physicochemical constant, $k$. The electrohydrodynamic parameters in subplots (a),(b) and (c) correspond to system-A, B and C, respectively. In both the figures a Mason number of $M=2$ is chosen.

From figures A-2(a) and (b) we observe that an increase in $\beta$ results in an enhanced gradient in surface tension $\left(\left|\gamma_{\max }-\gamma_{\min }\right|\right)$, but the deviations in the surfactant concentration from the equilibrium condition $\left(\left|\Gamma_{\max }-\Gamma_{\min }\right|\right)$ get suppressed with increasing $\beta$. This behavior is consistent with the work of Li \& Pozrikidis (1997) where the surfactant effects had been analyzed for the case of a drop in simple shear flow. Greater amount of surface tension gradient leads to an enhancement in Marangoni convection which in turn opposes the convective transport of surfactant. As a result the distribution of $\Gamma$ reaches towards equilibrium. An increase in $k$, on the other hand, signifies a corresponding increase in the convective transport mechanism of the surfactants (please refer to equation (11)). Thus for a fixed $\beta$, when $k$ is increased, the convective transport of surfactants dominates over the effect due to increased surface tension gradient, resulting in greater undulations in both $\Gamma$ and $\gamma$ from the equilibrium condition as portrayed in figures $\mathrm{A}-2$ (c) and (d), respectively. Now the Marangoni effect due to nonuniformity in surfactant distribution modifies the balance between the tangential component of the hydrodynamic and electrical Maxwell stresses at the interface (please refer to equation (9)).

In order to visualize the effect of surfactant-coating on the drop surface, in 4(a) to (d) we demonstrate the flow fields for some representative cases. These figures show that the orientation of the vorticity poles for system-B (figures $4(\mathrm{a})$, (b)) are vertical while it becomes horizontal for system-C (figures 4 (c), (d)). The surfactants create further alterations in both the velocity magnitude and the pattern of the disturbance flow around the drop. This subsequently causes an enhancement or reduction in the viscous dissipation of the bulk mixture and finally a corresponding change in the effective viscosity results.

The dependence of the normalized effective shear viscosity, $\left(\bar{\eta}_{\text {eff }}=\left(\eta_{\mathrm{eff}}^{(0)}-1\right) / \nu\right)$ on the shear rate is shown in figures $5(\mathrm{a})$ and $5(\mathrm{~b})$ for two different combinations of electrohydrodynamic parameters. First we define a relative hydrodynamic shear rate with respect to the electrical stress as $\bar{G}=1 / M=\frac{\mu_{c} \widetilde{G}}{\epsilon_{c} \widetilde{E}_{\infty}^{2}}$. It was previously reported Mandal \& Chakraborty, 2017b) that in the leading order, the effective viscosity becomes a function of relative shear rate $G$ only when the electric field is tilted with respect to direction of the applied shear flow, while the emulsion shows a Newtonian behavior when the electric field is applied in either along the direction of shear flow or its gradient. Now a careful inspection these figures reveals that the 


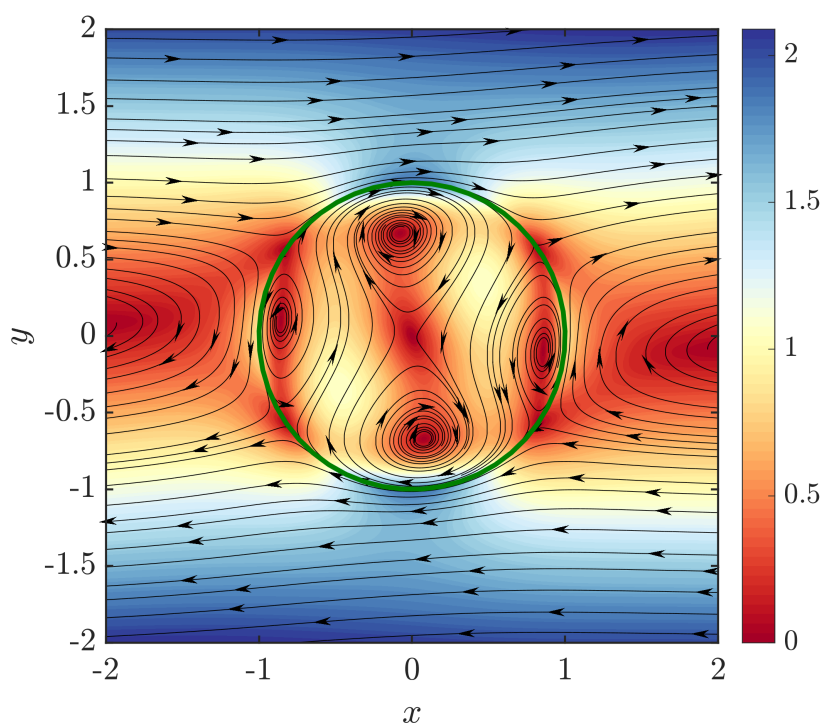

(a) Clean, system-B

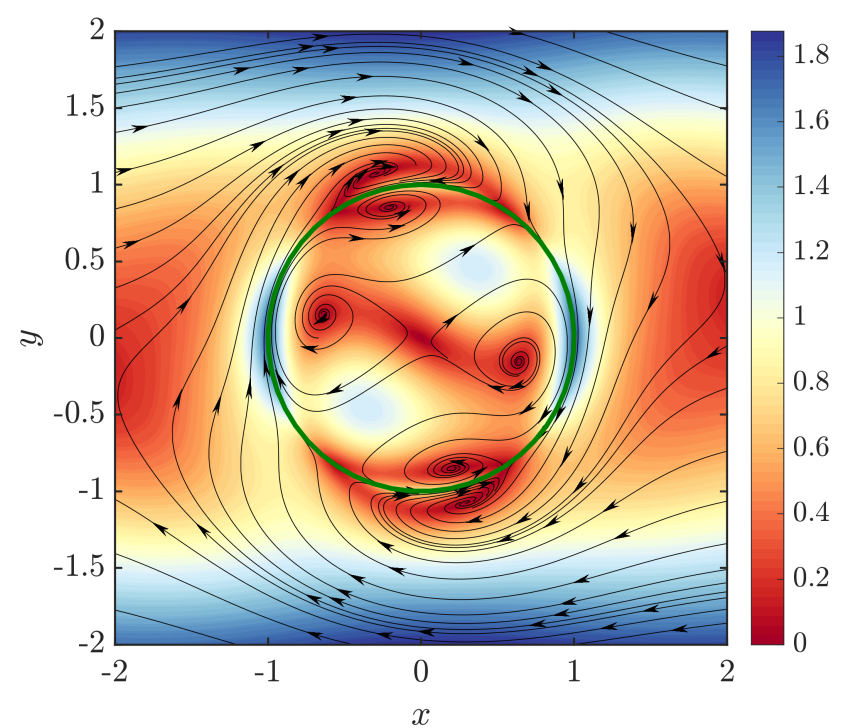

(c) Clean, system-C

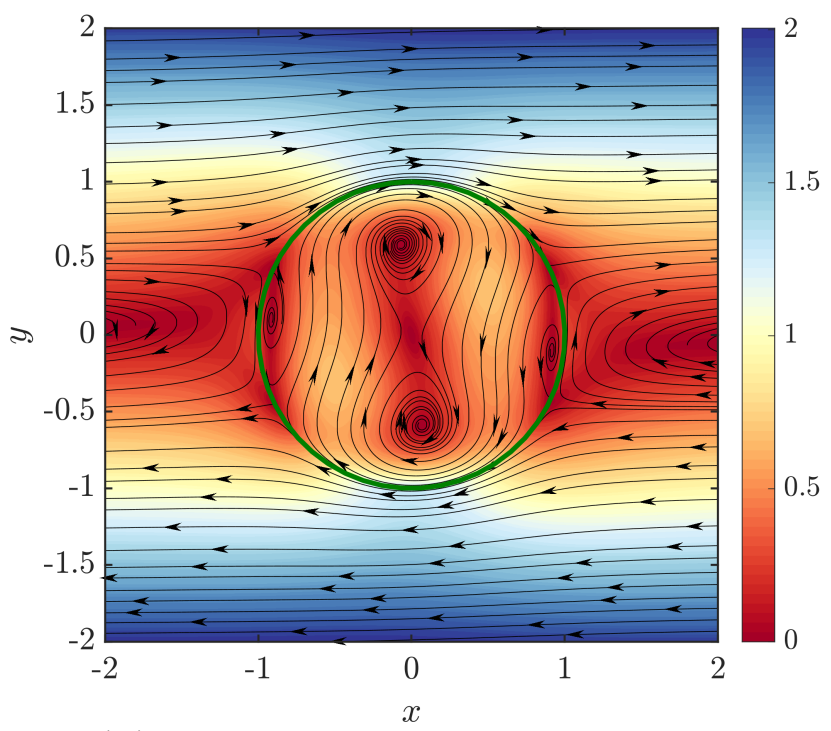

(b) Surfactant-coated, system-B

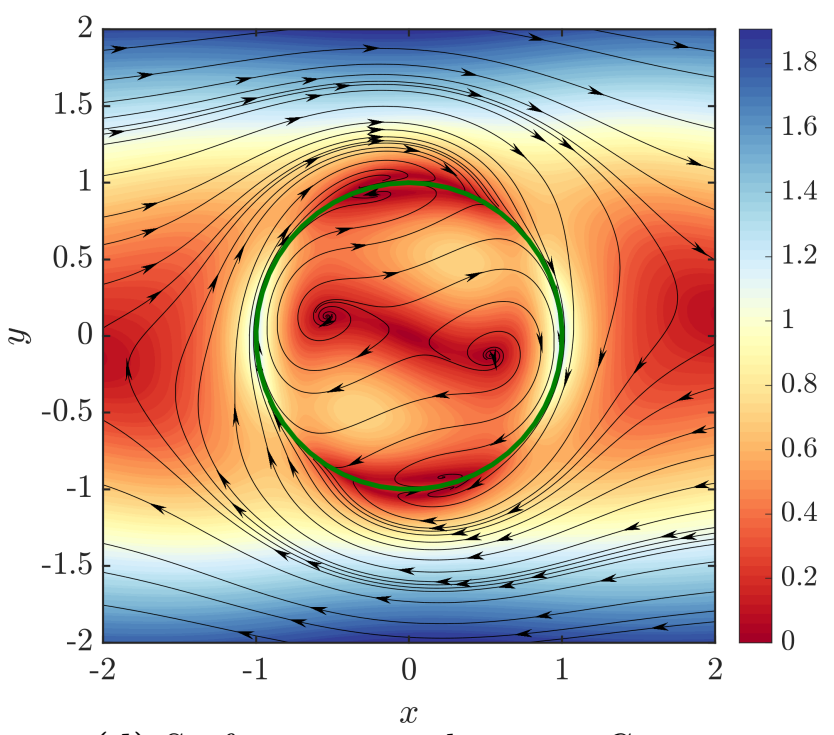

(d) Surfactant-coated, system-C

Figure 4: Comparison of leading order flow fields in and around a drop for clean and surfactantcoated case. The streamlines are shown in the plane of shear and with respect to a body-fitted reference frame. The color-bars show the variation in velocity magnitude, while the green circle in each figure denotes the drop-matrix interface. Subplots (a),(b) are for $M=5$, system-B and (c),(d) are for $M=5$, system-C. The parameters are chosen as $\Phi_{t}=\pi / 4, \beta=0.8$ and $k=1$.

non-uniformity surfactant distribution, has a potential in altering the non-Newtonian shear rate dependency of the effective shear rate. It is quite interesting to find from figure $5(\mathrm{a})$ that for a tilt angle of external electric field, $\Phi_{t}=\pi / 4$ there exists a crossover point at $\bar{G} \sim 0.2$ before which an increase in $\beta$ causes the effective shear viscosity to decrease in comparison to an uncontaminated drop, while beyond this point a corresponding increase in $\bar{\eta}_{\text {eff }}$ results with similar changes in $\beta$. However for $\Phi_{t}=3 \pi / 4, \bar{\eta}_{\text {eff }}$ shows a steady enhancement with $\beta$. In this case, for a fixed relative shear rate, the variation in $\beta$ can lead to a change in effective shear viscosity in such an extent that it becomes greater than the viscosity of the continuous medium $\bar{\eta}_{\text {eff }}>0$ or, $\mu_{\text {eff }}>\mu_{c}$, getting increased from a condition of $\bar{\eta}_{\text {eff }}<0$ or, $\mu_{\text {eff }}<\mu_{c}$ for a clean 
drop. The role of the electric field tilt angle gets reversed for system-B, as shown in figure 5(b). In the EHD literature the flow behavior is often shown to be of opposite nature when the electrical property combinations give rise to a positive $(R>S)$ or negative $(R<S)$ polarity (Taylor, 1966; Tsukada et al., 1993; Salipante \& Vlahovska, 2010; Mandal \& Chakraborty, $2017 b$ ). Based on the polarity, the direction of the electrical traction at the interface gets altered. However, comparing the present results in figure 3 for different electrohydrodynamic systems, it is observed that the consequence of the Marangoni effect cannot be simply categorized based on the condition of $R>S$ or $R<S$. Although system-A and B have properties $R<S$ and $R>S$, respectively, they exhibit similar trend of Marangoni effect, while both systems-A, C show opposite behavior despite having same polarity. Along similar lines, in figures 5(a) and $5(\mathrm{~b})$ the Marangoni effect is observed to be dependent on the electric field tilt angle $\left(\Phi_{t}\right)$ as well. Inspired by such observations we analyze the expression of the leading order effective shear viscosity, $\eta_{\mathrm{eff}}^{(0)}$ in equation 37 . We obtain a unique discriminating factor in terms of the crossover relative shear rate $\bar{G}_{\text {cr }}$ given as

$$
\bar{G}_{\mathrm{cr}}^{(0)}=\frac{9}{5} \frac{\sin \left(2 \Phi_{t}\right)(S-R)}{(R+2)^{2}}
$$

Similar a discriminating factors have been previously obtained by others in the context to drop deformation (Taylor, 1966) and surface charge convection $\mathrm{Xu} \&$ Homsy, 2006). If $\Phi_{t}<\frac{\pi}{2}$ then $\sin \left(2 \Phi_{t}\right)$ is a positive quantity. Thus for a realistic $\bar{G}_{\mathrm{cr}}^{(0)}$ to exist, the condition which must be satisfied is $R<S$. Again for $\Phi_{t}>\frac{\pi}{2}$ the property ratios must fulfill the condition $R>S$. Hence the Marangoni effect on the electrically induced emulsion viscosity can be suitably controlled by finely tuning the combination of the electric field direction and electrical properties of the dropsurrounding fluid pair. To understand such a behavior of Marangoni effect we plot the surface tension gradient $\left(\left|\gamma_{\max }-\gamma_{\min }\right|\right)$ with relative shear rate for different values of the tilt angle in figure 5(c) for system-A. It shows that the surface tension gradient vanishes at a relative shear rate which is exactly equal to the crossover point observed in figure 5(a). This indicates that due to competitive effects of the hydrodynamic and electrical stresses on the surface tension variation, for a specific relative shear rate, the Marangoni effect vanishes completely and the drop surface behaves as an uncontaminated one. The corresponding competing effects can be realized by referring back to equation (37) where distinct functional forms of the hydrodynamic and electric surfactant contribution terms $\left(\eta_{\mathrm{h}, \text { surf }}^{(0)}, \eta_{\mathrm{e}, \text { surf }}^{(0)}\right)$ appeared in the expression of $\eta_{\mathrm{eff}}^{(0)}$. The lesser extent of variation of $\eta_{\text {eff }}^{(0)}$ with $\bar{G}$, after the crossover point $\bar{G}_{c r}$ is reached (observation from figure 5(a) , can also be explained from figure $5(\mathrm{c})$ where the range of surface tension gradient $\left(\left|\gamma_{\max }-\gamma_{\min }\right|\right)$ becomes lower after $\bar{G}_{c r}$ than it was before $\bar{G}_{c r}$.

The value range of $\bar{G}_{c r}^{(0)}$ is observed to be different in the two set of electrohydrodynamic parameters. In this context, the parametric mapping of the critical relative shear rate on the $R-S$ plane is shown for two different tilt angles in figures $5(\mathrm{~d})$ and $5(\mathrm{e})$, respectively. It is to be noted that a critical normalized shear rate was obtained previously by Mandal \& Chakraborty $(2017 b)$ in relation to a surfactant-free drop under the combined influence of electric field and shear flow. In that case, the critical parameter had been used to characterize whether the effective shear viscosity is greater (or lesser) than the suspending medium. In stark contrast, the presently obtained critical relative shear rate is related to the surfactant induced Marangoni effect. To the best of our knowledge, for the first time we have obtained a discriminating factor 
in terms of a relative shear rate for which the Marangoni effect will have no contribution in altering the emulsion rheology.

The another important aspect of bulk rheology, i.e. the first $\left(N_{1}^{(0)}\right)$ and second $\left(N_{2}^{((0))}\right)$ normal stress differences are significantly affected by the presence of surfactant contamination on the drop surface. Equation (38) shows that in the leading order the normal stress differences in the bulk rheology arise only from the electrohydrodynamic origins even when the surfactant effects are considered. From the functional form of the $\mathcal{N}_{\text {surf }}^{(0)}$ it is evident that for all the realistic parametric ranges of the parameters $(0 \leq \beta<1, k>0$ and $\lambda>0)$ the surfactant induced Marangoni effect always acts to reduce the normal stress differences, i.e. $\mathcal{N}_{\text {surf }}^{(0)}<1$. In figure 6 a parametric map of the common surfactant contribution factor $\mathcal{N}_{\text {surf }}^{(0)}$ (defined in equation (39p) is shown on $\beta-k$ plane for different viscosity ratios of the drop-matrix fluid pair. It shows that for lower viscosity of the drop phase as compared to the matrix fluid, the surfactant contribution is more prominently observed. In the limit of $\lambda \rightarrow 0$, when the drop tends behave as a bubble, the surfactant effect is the maximum and we obtain $\lim _{\lambda \rightarrow 0} \mathcal{N}_{\text {surf }}^{(0)}=\frac{(1-\beta)}{(k-5) \beta+5}$. On the other limit of $\lambda \rightarrow \infty$, the drop behaves like a particle and its surface becomes completely immobilized. In that case the normal stress differences vanish themselves for a spherical drop.

\subsection{Interplay between charge convection and surfactant transport}

For a clean drop, the presence of finite charge convection creates a redistribution of charges. Following the discussion in section 3.5, the $O\left(R e_{E}\right)$ electrical potential and in turn the accumulated surface charge density become dependent on the leading order interfacial fluid velocity $\left(\mathbf{u}_{S}^{(0)}\right)$, which is already affected by the surfactant effects (please refer to equation A-1 for full expression). In figure 7(a) we demonstrate the variations in the surface charge distribution $\left(q_{S}=q_{S}^{(0)}+R e_{E} q_{S}^{\left(R e_{E}\right)}\right)$ for different surface elasticity parameter $\beta$. It is observed that for a surfactant-contaminated drop, the redistribution of charges due to the presence of finite charge convection at the interface is affected by the Marangoni effects. On the other hand, the modulations in the electrohydrodynamic flow created by the finite charge convection, interacts with the Marangoni convection and variations in the surface tension result. This behavior is shown in figure $7(\mathrm{~b})$.

\subsubsection{Order $\operatorname{Re}_{E}$ bulk rheology}

The coupled effects of charge convection and surfactant non-uniformity is described in figure 8 . Figure 8(a) depicts than increase in the parameter $\beta$ suppresses the $O\left(R e_{E}\right)$ modifications in $\bar{\eta}_{\text {eff }}$. To justify such phenomena we plot the variations in $O\left(R e_{E}\right)$ surface velocity and the electrical traction at the interface in figure A-3 for different values of $\beta$. The figure shows that the charge convection triggered modifications in the flow characteristics (exemplified by modulations in

$u_{S, \phi}^{\left(R e_{E}\right)}$ and $\left.\left[\left[T_{\phi}^{E}\right]\right]^{\left(R e_{E}\right)}\right)$ are severely altered due to the surface elasticity parameter, $\beta$.

The above interplay between the charge convection and Marangoni flow at the interface, also modifies the shear rate dependent response of the emulsion viscosity, as shown in figure 8 . The critical relative shear rate corresponding to the crossover point of the Marangoni effect $\left(\bar{G}_{c r}\right)$, gets shifted from its leading order counterpart (shown in figure $8(\mathrm{a})$ ). This effect can be substantiated by carefully inspecting the $O\left(R e_{E}\right)$ effective shear viscosity $\left(\eta_{\mathrm{eff}}^{\left(R e_{E}\right)}\right)$. Similar to 


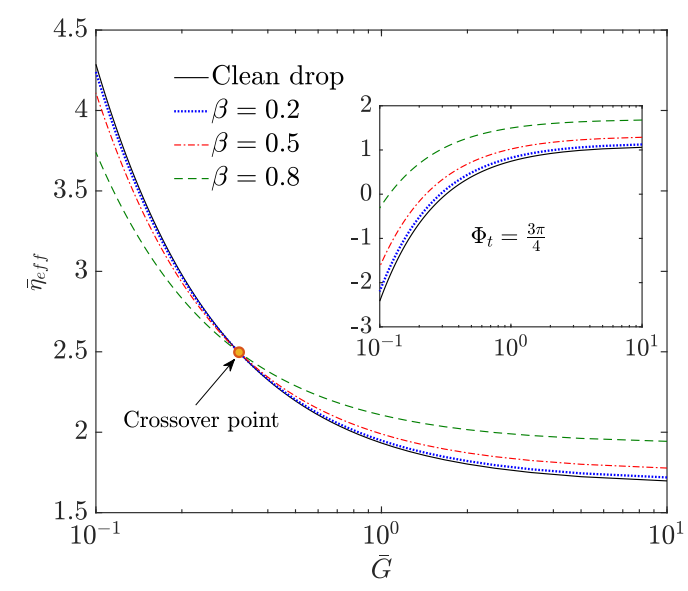

(a) System-A

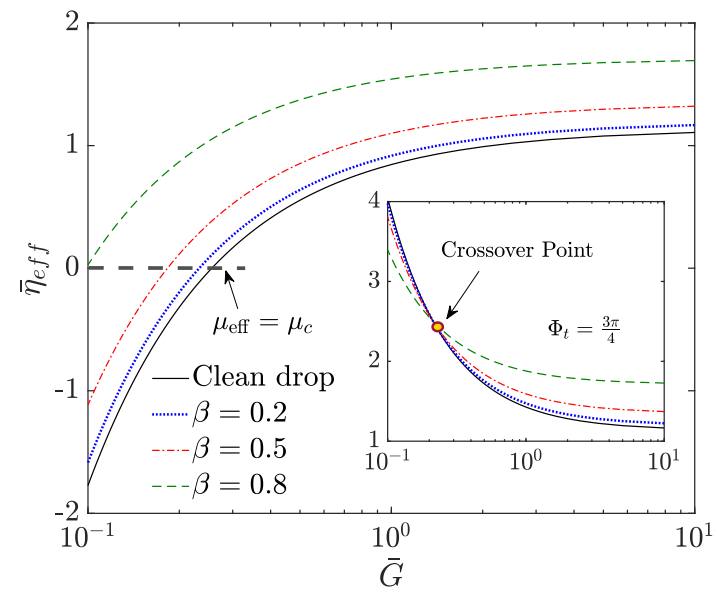

(b) System-B

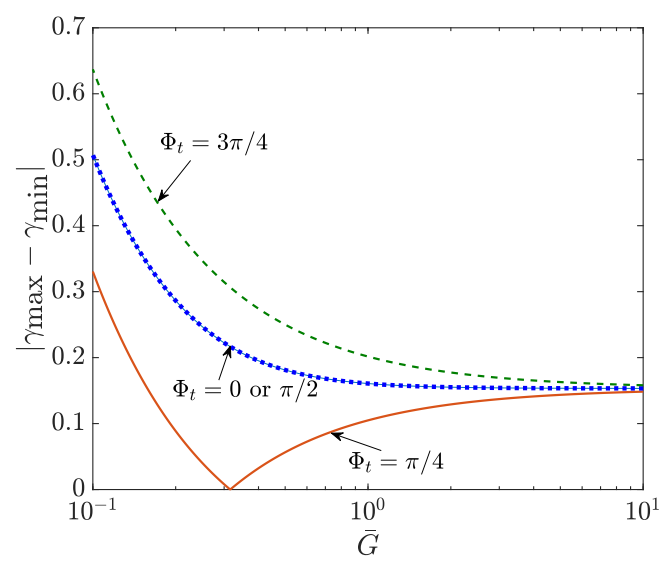

(c)

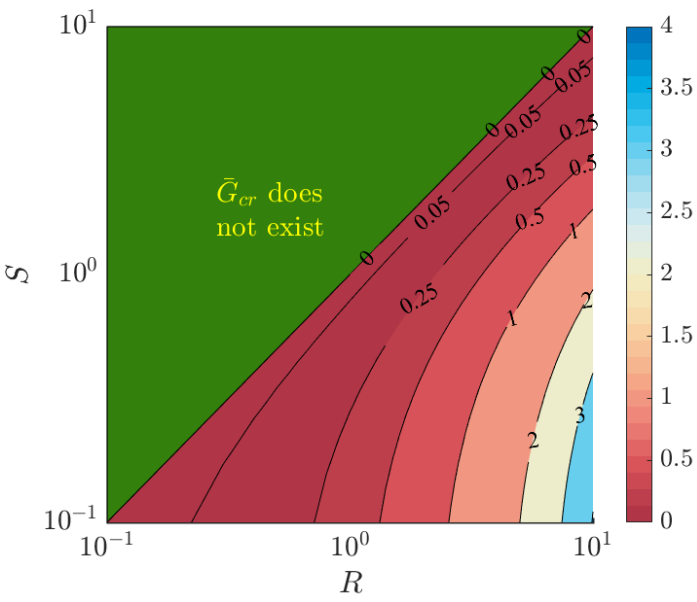

(d) $\Phi_{t}=\pi / 4$

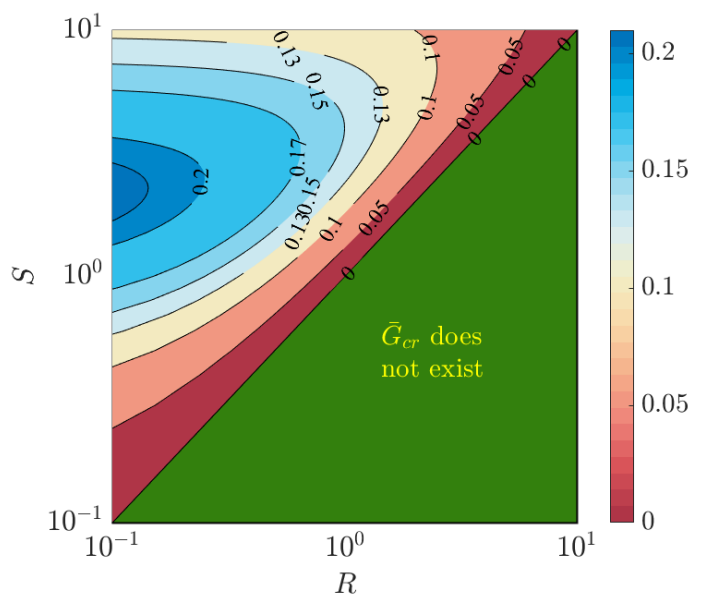

(e) $\Phi_{t}=3 \pi / 4$

Figure 5: Subplot (a) and (b): Variation of normalized effective shear viscosity $\left(\bar{\eta}_{\text {eff }}\right)$ with relative shear rate $\bar{G}=1 / M$ for different values of the elasticity parameter, $\beta$. The subplots (a) and (b) are for system-A and B, respectively. In both the subplots $\Phi_{t}=\pi / 4$ is chosen, while the inset to each subplot shows the corresponding variations for $\Phi_{t}=3 \pi / 4$. Subplot (c): Variation of surface tension gradient $\left(\left|\gamma_{\max }-\gamma_{\min }\right|\right)$ with relative shear rate for various $\Phi_{t}$. Here the demonstration is for system-A. Subplot (d) and (e) show the maps of the critical relative shear rate on the $(R, S)$ plane for electric field tilt angle, $\Phi_{t}=\pi / 4$ and $\Phi_{t}=3 \pi / 4$, respectively. 

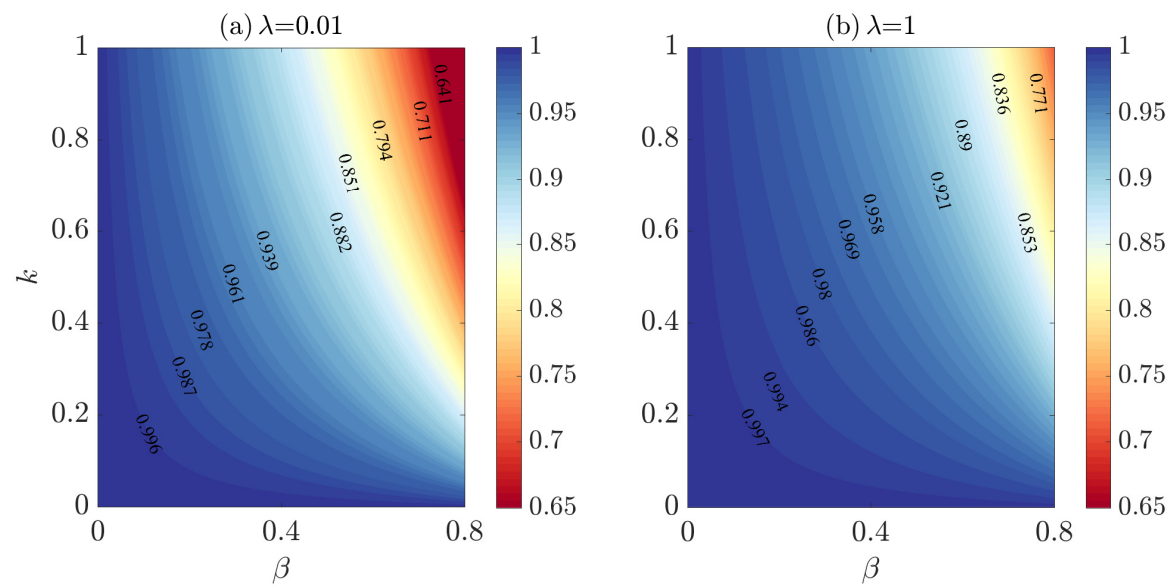

Figure 6: Surfactant modification parameter for the normal stress differences of the emulsion $\left(\mathcal{N}^{(0)}\right)$ on $\beta-k$ plane for different viscosity ratios $(\lambda)$.

the leading order, there exists a specific range of relative shear rate for which the Marangoni effect has hardly any role in modifying the $O\left(R e_{E}\right)$ effective viscosity of the medium $\left(\eta_{\mathrm{eff}}^{\left(R e_{E}\right)}\right)$. However the critical shear rate in this case is also a function of the surfactant parameters and the viscosity ratio, as shown below:

$$
\begin{gathered}
\bar{G}_{\mathrm{cr}, \bar{\eta}}^{\left(R e_{E}\right)}=\frac{R-S}{(R+2)^{2}} \frac{\sin \left(2 \Phi_{t}\right)}{\delta_{1} \cos \left(2 \Phi_{t}\right)+\delta_{2}}, \text { where } \\
\delta_{1}=\frac{175}{36} \frac{(3 R+4)(\lambda+1)(R-2 S-2)(\beta k-5 \beta \lambda-5 \beta+5 \lambda+5)}{\left(69 R^{2}-54 R S+130 R-80 S+40\right)(\beta k-10 \beta \lambda-10 \beta+10 \lambda+10)}
\end{gathered}
$$

and

$$
\delta_{2}=-\frac{5}{108} \frac{\left(701 R^{2}-506 R S+1410 R-760 S+520\right)}{\left(69 R^{2}-54 R S+130 R-80 S+40\right)} .
$$

In addition, comparing figures $8(\mathrm{a})$ and (b), we observe that the non-Newtonian shear thickening (shear thinning) rheology at low shear rates (corresponding to the curve $R e_{E}=0.2$, clean drop), becomes a shear thinning (shear thickening) one in the presence of surfactant (refer to the curve $R e_{E}=0.2, \beta=0.8$ ). This reversal in non-Newtonian behavior is absent when charge convection effects are insignificant.

In the absence of charge convection, the surfactant contributions to the first and second normal stress differences $\left(\Delta \bar{N}_{1}=\left|\bar{N}_{1}-\bar{N}_{1 \text {,clean }}\right|, \Delta \bar{N}_{2}=\left|\bar{N}_{2}-\bar{N}_{2, \text { clean }}\right|\right)$ increase with the tilt angle and are symmetric about $\Phi_{t}=\pi / 2$, as depicted in figures 9(a) and 9(b). $\Delta \bar{N}_{1}$ takes a maximum value for $\Phi_{t}=0, \pi / 2$ and vanishes for $\Phi_{t}=\pi / 4$; while $\Delta \bar{N}_{1}$ increases from zero to maximum from $\Phi_{t}=0$ to $\pi / 2$. However, owing to the presence of the charge convection, such symmetric nature is broken, although the increasing trends of both $\Delta \bar{N}_{1}$ and $\Delta \bar{N}_{2}$ are preserved.

\subsection{Bulk rheology of deformed drops}

Here we analyze the consequences of the coupled interaction between the deformable nature of the drop and surfactant coating for different electrohydrodynamic systems. From figures 10(a) and (b) it can be observed that the deformation induced modification in the Marangoni effect 


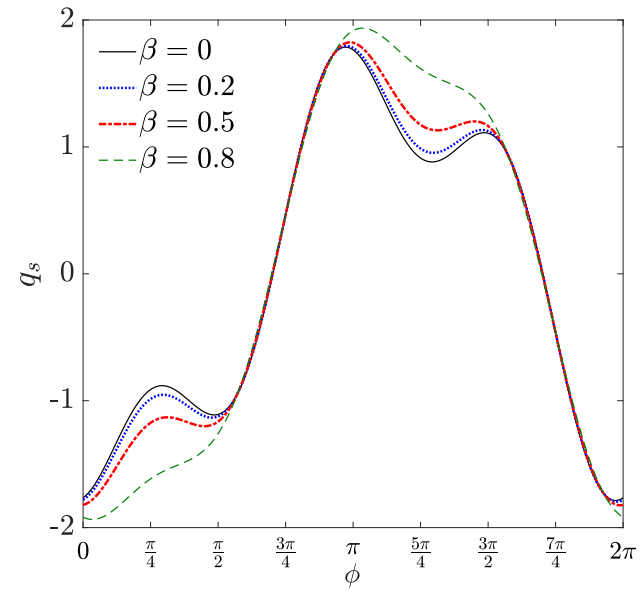

(a)

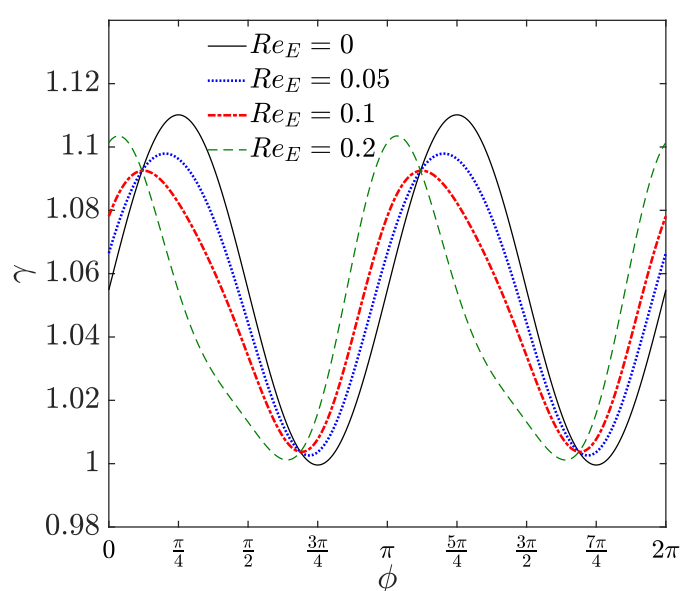

(b)

Figure 7: (a) Azimuthal variation of the surface charge density $\left(q_{S}=q_{S}^{(0)}+R e_{E} q_{S}^{\left(R e_{E}\right)}\right)$ for different values of the surface elasticity parameter, $\beta$. (b) variation of the surface tension $\left(\gamma=\gamma^{(0)}+C a \gamma^{(C a)}+C a R e_{E} \gamma^{\left(R e_{E}\right)}\right)$ for different values of electric Reynolds number $\left(R e_{E}\right)$. The demonstrations are for system-C. Parameters are chosen as $R e_{E}=0.2, M=2, k=1, \Phi_{t}=\pi / 4$ and $C a=0.2$.

is much pronounced for lower values of the elasticity parameter $\beta$. Also the effect of capillary number becomes smaller as $\beta \rightarrow 0.8$. Most interestingly, the increase in capillary number causes a fall in the effective viscosity for system-B, while the trend is opposite for system-C. To understand the physical origin of such behavior we investigate the nature of drop deformation in different systems and the consequence of surfactant coating on them. For this we appeal to the normal stress balance equation (10) where the Marangoni effect is seen to play its role. From physical perspective, the localized accumulation of surfactant molecules reduces the local surface tension and makes that region more prone to deformation in order to balance the normal stresses. This is known as the 'tip stretching' phenomena in the literature (Pawar \& Stebe, 1996). Again due to increased surface area of the deformed drop, the overall surfactant concentration gets diluted and the local surface tension tends to fall.

In order to quantify the deformation behavior of the drop we define a deformation parameter in the plane of shear as follows,

$$
\mathcal{D}=\frac{\max \left(r_{S}\left(\theta=\frac{\pi}{2}, \phi\right)\right)-\min \left(r_{S}\left(\theta=\frac{\pi}{2}, \phi\right)\right)}{\max \left(r_{S}\left(\theta=\frac{\pi}{2}, \phi\right)\right)+\min \left(r_{S}\left(\theta=\frac{\pi}{2}, \phi\right)\right)} .
$$

Another important quantification of the drop deformation is the drop inclination angle which can be defined in the $x-y$ plane as the angle $\phi$ corresponding to the maximum value of $r_{S}$ for $\theta=\pi / 2$ and is given as $\varphi_{d}=\frac{1}{2} \tan ^{-1}\left(\hat{L}_{2,2} / L_{2,2}\right)$. In comparison to the case when only a simple shear flow is present (Li \& Pozrikidis, 1997), the electrical effects (with $\Phi_{t}=\pi / 4, M=2$ ) increases the deformation parameter $(\mathcal{D})$ for system-B. In contrast, for system-C the initial prolate shape (with respect to the applied electric field direction along $\Phi_{t}=\pi / 4$ ) of the drop under simple shear flow becomes a complete oblate one when electrical effects are present. This phenomenon is portrayed in the insets of the figures A-4(a) and (b)). This condition for a surfactant-free drop surface is further modified by surface contamination and the deformation parameter shows a steady increase with $\beta$ as shown in figures $A-4$ (a) and (b). Thus the nature 


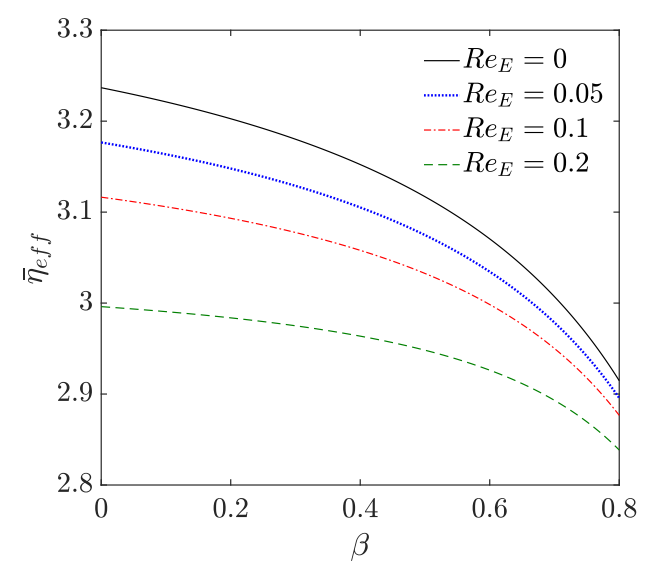

(a)

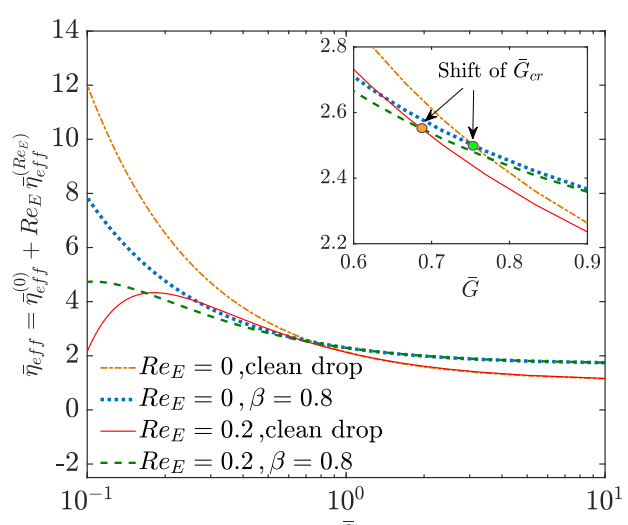

(b) $\Phi_{t} \stackrel{\bar{G}}{=} \pi / 4$

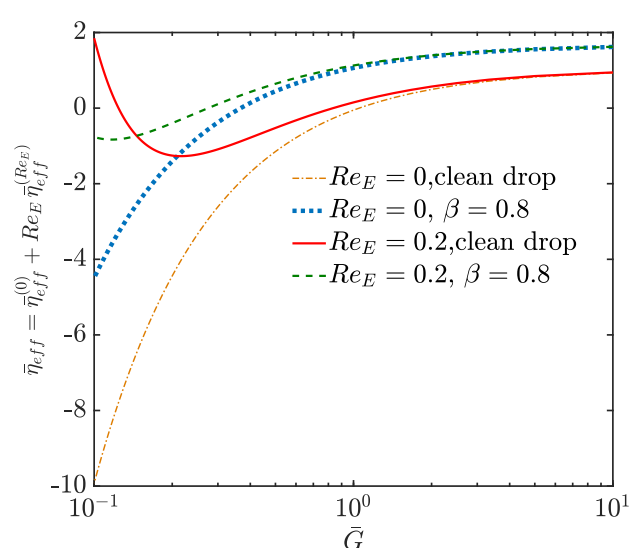

(c) $\Phi_{t} \stackrel{G}{=} 3 \pi / 4$

Figure 8: (a): Normalized effective shear viscosity $\left(\bar{\eta}_{\text {eff }}=\bar{\eta}_{\text {eff }}^{(0)}+R e_{E} \bar{\eta}_{\text {eff }}^{\left(R e_{E}\right)}\right)$ variation with elasticity parameter $\beta$ for different electric Reynolds number. Here $M=2$ and $\Phi_{t}=\pi / 4$. (b),(c): $\bar{\eta}_{\text {eff }}$ variation with relative shear rate, $(\bar{G})$ for different elasticity parameter, $\beta$ and electric Reynolds number, $R e_{E}$. In the inset of subplot (b) the shift of critical shear rate, $\bar{G}_{\mathrm{cr}}$ with $R e_{E}$ is highlighted. Here $\Phi_{t}=\pi / 4$ and $\Phi_{t}=3 \pi / 4$ in subplots (b) and (c), respectively. The other parameters in all the sub-figures are chosen following system-C.

as well as intensity of drop deformation is modified. As a result the drop poses different kinds of resistances to the imposed shear flow, thereby creating diverse modulations in the effective shear viscosity of the emulsion.

The deformation of the drop surface also influences the non-Newtonian rheology of the emulsion as shown in figure 10(c). The critical shear rate corresponding to the vanishing Marangoni effect $\left(\bar{G}_{c r}\right)$ gets shifted to a higher value when the capillary number becomes 0.2 from an undeformed state, for the specific parametric choices of system-C. Till $\bar{G}_{c r}$ is reached, the shape deformation causes significant enhancement in the effective shear viscosity. However, with an increase in $\bar{G}$ beyond this critical point, the impact of deformation effects on the effective viscosity, becomes vanishingly small.

In figure 11 we demonstrate the variations in the bulk normal stress differences with the tilt angle of the applied electric field for different values of the surface elasticity number and capillary number. It is found that the leading order symmetry in both $\bar{N}_{1}$ and $\bar{N}_{2}$ about the tilt angle $\Phi_{t}=\pi / 2$ is destroyed owing to a deformed drop surface. In the deformed 


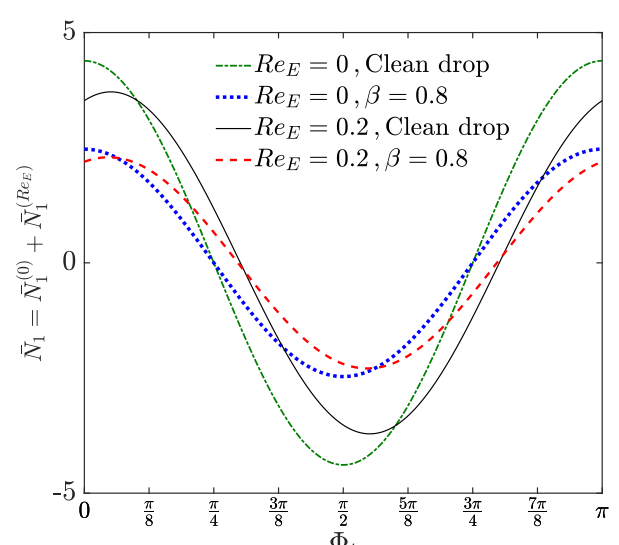

(a)

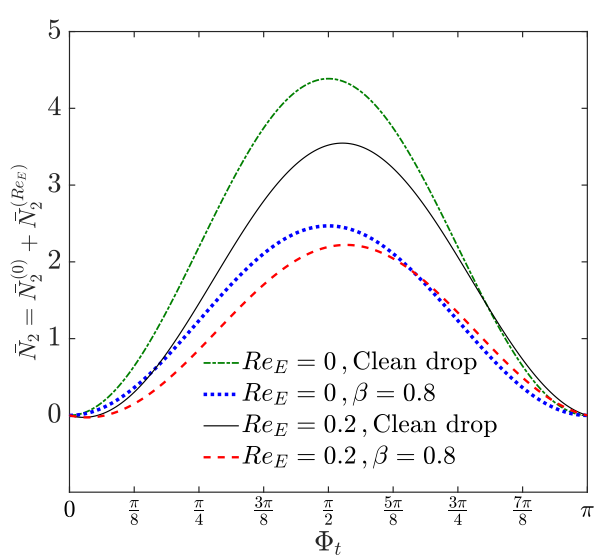

(b)

Figure 9: Variation of normalized first $\left(\bar{N}_{1}=N_{1} / \nu\right)$ and second $\left(\bar{N}_{2}=N_{2} / \nu\right)$ normal stress differences in subplots (a) and (b), respectively with the tilt angle of the applied electric field, $\Phi_{t}$ for different values of elasticity parameter $\beta$ and electrical Reynolds number, $R e_{E}$. Here the electrohydrodynamic parameters are chosen according to system-C. Other parameters are $M=2$ and $k=1$.

condition, the contribution of the surfactants in modifying the first normal stress difference $\left(\Delta \bar{N}_{1}=\left|\bar{N}_{1}-\bar{N}_{1, \text { clean }}\right|\right)$, is observed to be positive for certain values of the tilt angle, while the reverse happens for other tilt angles (please refer to figure 11(a)). However, figure 11(b) depicts that under similar circumstances, the surfactant-induced modifications in the second normal stress difference $\left(\Delta \bar{N}_{2}=\left|\bar{N}_{2}-\bar{N}_{2 \text {,clean }}\right|\right)$ show a steady decrease for all the tilt angles between $\Phi_{t}=0$ and $\pi$. Variations in the orientation of the applied electric field not only alters the behavior of the drop deformation but also cause severe changes the electrohydrodynamic flow pattern around the drop. In turn, the surface tension gradient is redistributed. Finally, an intricate interplay between the physical mechanisms of electrohydrodynamic and Marangoni flow dictates the elastic characteristics of the emulsion, resulting in corresponding changes in the normal stress differences.

\section{Conclusions}

In the above sections we presented a detailed analysis of the surfactant coating effects on the electrorheology of a dilute emulsion. We have considered a linear shear flow and a tilted electric field acting in tandem on a deformable viscous drop which is covered with non-ionic, bulk insoluble surfactants. The electrical effects have been modeled through the Taylor-Melcher leaky dielectric framework. Also the surfactant transport at the interface is considered to be diffusion dominated and the equations are obtained in the limit of small surface Peclet number, $P e_{S}$. Next we have employed a double asymptotic perturbation in terms of small dimensional parameters of electrical Reynolds number $\left(R e_{E}\right)$ and capillary number $(\mathrm{Ca})$. In the absence of drop deformation, the tangential stress balance at the interface (equation (9)) is interacted by the Marangoni stresses at the interface due to non-uniform surfactant distribution. This creates alterations in the tangential flow around the drop and subsequently the nature of viscous dissipation due to the presence of the drops is changed. On the other hand, the drop deformation is also severely influenced by the Marangoni stresses (equation 10 ), and finally the drop shape 


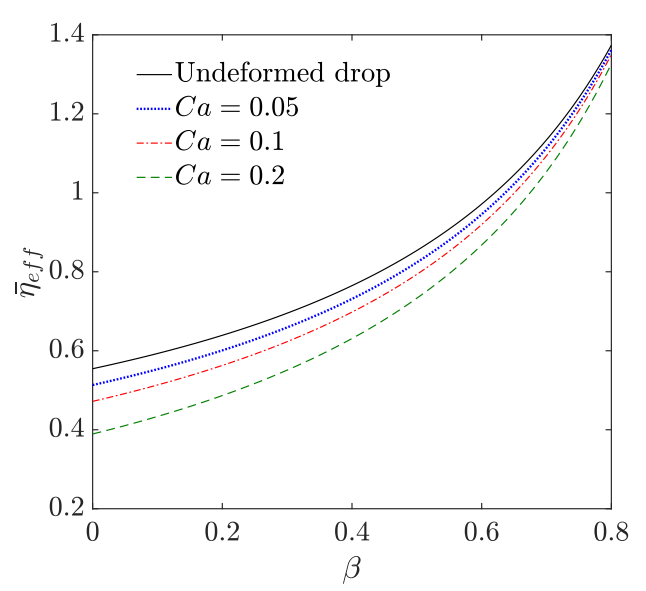

(a) System-B

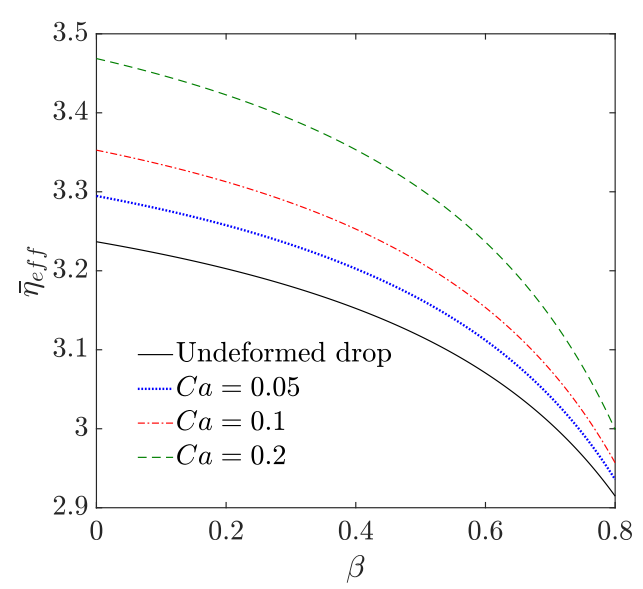

(b) System-C

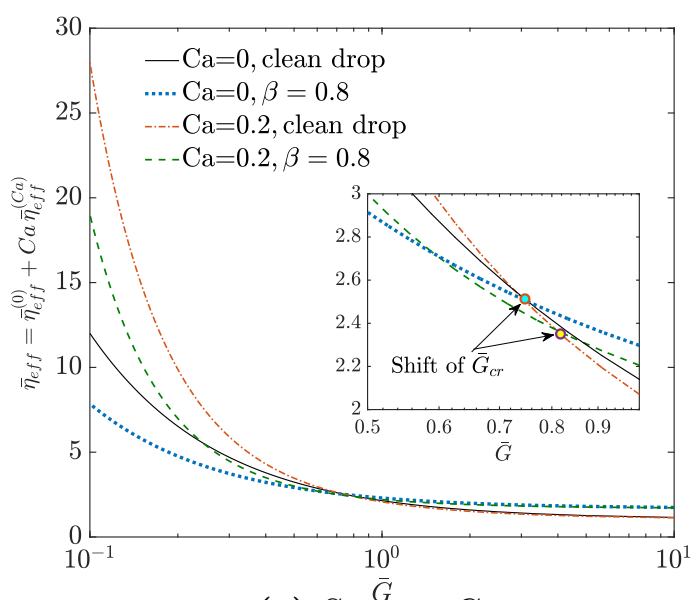

(c) System-C

Figure 10: (a),(b): Normalized effective shear viscosity $\left(\bar{\eta}_{\text {eff }}=\bar{\eta}_{\text {eff }}^{(0)}+C a \bar{\eta}_{\text {eff }}^{(C a)}\right)$ vs. elasticity parameter, $\beta$ for different capillary numbers, $C a$. The subplots (a) and (b) are for system-B and $\mathrm{C}$, respectively. Here $M=2$. Subplot-(c): Normalized effective shear viscosity vs. relative shear rate for different elasticity parameter, $\beta$ and Capillary number, $C a$. In the inset, the shift of critical shear rate, $\bar{G}_{\text {cr }}$ with $C a$, is highlighted. Here other parameters are chosen following system-C. In all the subplots $\Phi_{t}=\pi / 4$ and $k=1$.

is distorted in a varying extent. The deformation of the drop in or against the flow direction, can make the emulsion shear thinning or shear thickening, respectively. Not only the viscosity, but also the effective tension along the flow streamlines, is affected by the rich physical interaction of the Marangoni, hydrodynamic and electrical Maxwell stresses. We can conclude the below mentioned points from the presented work:

(i) The surfactant distribution on the drop surface not only depends on the imposed shear flow, but also the tilt angle of the applied field plays a major role in dictating the same. In some cases, this modification is so severe that the peak surfactant accumulation along the poles of the straining axis in the plane of shear, is changed to out of the plane locations. The relative intensity of the hydrodynamic and electrical stresses, quantified by the Mason number, determines the points of peak surfactant accumulation and in turn, the nature of surface tension gradient $\left(\left|\gamma_{\max }-\gamma_{\min }\right|\right)$ on the interface is also influenced.

(ii) Both the hydrodynamic and electrical components of the effective shear viscosity are 


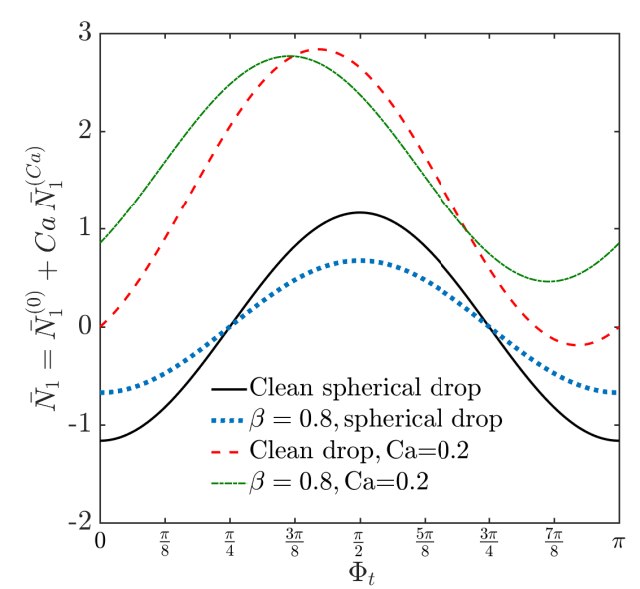

(a)

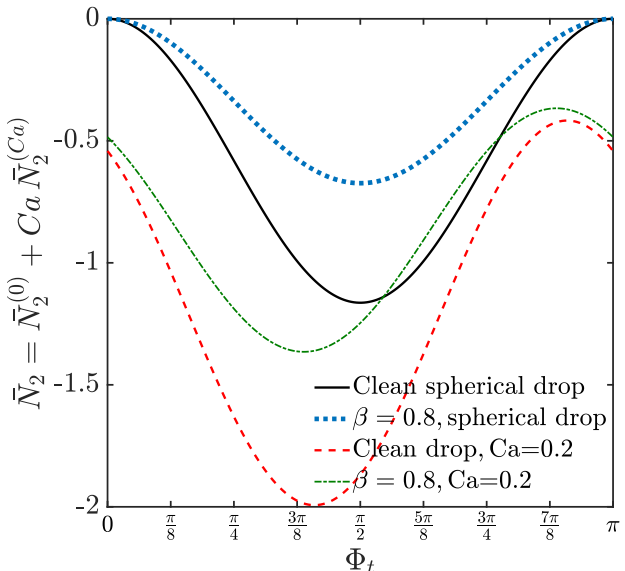

(b)

Figure 11: Normalized first $\left(\bar{N}_{1}=N_{1} / \nu\right)$ and second $\left(\bar{N}_{2}=N_{2} / \nu\right)$ normal stress differences vs. tilt angle of the applied electric field, $\Phi_{t}$ for various elasticity parameter $\beta$ and Capillary number, $\mathrm{Ca}$. Here the electrohydrodynamic parameters are chosen according to system-B. Other parameters are $M=2$ and $k=1$.

modulated greatly due to surface contamination, beyond just a linear superposition to the existing expressions for a clean drop. Depending on the drop-matrix combination of the electrohydrodynamic parameters, the overall surfactant contribution can either increase or decrease the effective shear viscosity.

(iii) We report the unique existence of a critical hydrodynamic shear rate relative to the electric stress for which the surface tension gradient vanishes on the drop surface. At this critical relative shear rate, the corresponding surfactant effect on the effective shear viscosity, gets vanished. This critical shear rate strongly depends on the tilt angle of the applied electric field,the electrical conductivity ratio and the permittivity ratio of the drop-matrix fluid pair.

(iv) The bulk normal stress differences, describing the anisotropy in the normal stress of the effective fluid mixture, always reduce due to surfactant effect in the leading order. This decrement becomes much pronounced for low drop viscosities.

(v) The interaction between surfactant-induced Marangoni stress and the surface charge convection, can make the shear response of the emulsion, from shear thinning to shear thickening or vice-versa, depending on the tilt angle of electric field. The charge convection also creates a shift in the critical shear rate for vanishing surfactant effect, as observed in the leading order.

(vi) In the deformed condition, for a specific choice of the electrohydrodynamic parameters, the drop shape may become ellipsoidal along the direction of the applied electric field or perpendicular to it. Now the surfactant effects cause the deformation intensity to rise in both cases. Finally the resistance to flow is affected in such a way that the deformation-induced rise or fall in the effective shear viscosity, gets suppressed due to Marangoni effects. Similar to the charge convection, the shape deformation can also create a shift in the critical shear rate.

To summarize, the detailed analysis presented above shows that the disturbance in dropscale flow dynamics, created due to the presence of dispersed drops in a combined action of shear flow and uniform electric field, can be severely modulated by surfactant coating on the interface. 
As a consequence, the bulk rheological properties of a dilute emulsion may exhibit distinct nonNewtonian behaviors depending upon the highly coupled interaction between the surfactant distribution on the interface and electrohydrodynamic flow pattern. The understanding of the micro-scale flow physics, depicted by the above results and its impact on the rheology, thus become a stepping stone towards analyzing more complex rheological behavior of emulsions where multi-drop interactions become important.

\section{Appendix A. Detailed expression of the leading order surface velocity $\mathbf{u}_{S}^{(0)}$}

The leading order surface velocity can be expressed in the spherical coordinate system as $\mathbf{u}_{S}^{(0)}=u_{S, \theta}^{(0)} \mathbf{i}_{\theta}+u_{S, \phi}^{(0)} \mathbf{i}_{\phi}$, where the scalar components are of the form:

$$
\begin{aligned}
u_{S, \theta}^{(0)}= & \frac{\left\{\begin{array}{c}
(1-\beta)\left(\left(9 M(R-S) \sin \left(2 \Phi_{t}\right)+5(2+R)^{2}\right) \sin (2 \phi)\right. \\
+9 M\left(1+\cos \left(2 \Phi_{t}\right) \cos (2 \phi)\right)
\end{array}\right\} \sin (2 \theta)}{4(R+2)^{2}(k \beta-5 \beta \lambda-5 \beta+5 \lambda+5)}, \text { and } \\
u_{S, \phi}^{(0)}= & \frac{\sin (\theta)}{((k-5 \lambda-5) \beta+5 \lambda+5)(2+R)^{2}}\left\{\left(\frac{9 M}{2}(R-S)(\beta-1) \sin \left(2\left(\Phi_{t}-\phi\right)\right)\right.\right. \\
& \left.\left.+\frac{5(2+R)^{2}(\beta-1) \cos (2 \phi)}{2}\right)-\left(\frac{1}{2}(k-5 \lambda-5) \beta+\frac{5 \lambda}{2}+\frac{5}{2}\right)(2+R)^{2}\right\} .
\end{aligned}
$$

\section{Appendix B. Comparison with previous works}

In different steps of calculation, the expressions of important physical variables have been validated against various limiting cases of the present physical situation, as elaborated below:

(i) Surfactant-free drop in shear flow:

This simplistic case can be obtained in the present study by substituting $M=0$ and $\beta=0$ in the resulting expressions. Under such a condition the leading order effective shear viscosity was first obtained by Taylor (1932). Our leading order results in equation (37), i.e. $\eta_{\mathrm{h}, \text { clean }}^{(0)}=$ $1+\nu \frac{(5 \lambda+2)}{(2 \lambda+2)}$, corroborates with that classic result.

In the same limit, we obtained the $O(C a)$ normal stress differences as given below:

$$
N_{1}^{(C a)}=\nu \frac{(16+19 \lambda)^{2}}{40(\lambda+1)^{2}}
$$

and

$$
N_{2}^{(C a)}=-\nu \frac{551 \lambda^{3}+1623 \lambda^{2}+1926 \lambda+800}{280(\lambda+1)^{3}} .
$$

Also in $O(\mathrm{Ca})$, the hydrodynamic contribution to the effective shear viscosity is obtained as zero $\left(\eta_{\text {eff }}^{(\mathrm{Ca})}=0\right)$. These observations are in agreement with the seminal work of Schowalter et al. (1968). For similar conditions, the $O(C a)$ shape function $f^{(\mathrm{Ca})}$ matches with the expressions obtained by Barths-Biesel \& Acrivos (1973) and Vlahovska (2011), respectively.

(ii) Surfactant-coated drop in shear flow:

The expressions in the vanishing diffusion regime or the high surface Péclet number regime 
$\left(P e_{S} \gg 1\right)$ (Vlahovska et al., 2009) can be obtained by taking a limit $k \rightarrow \infty$ and substituting $M=0$ in the present expressions, as shown below:

$$
N_{1}^{(C a)}=\frac{5 \nu(3 \beta+1)}{2 \beta}
$$

and

$$
N_{2}^{(C a)}=-\frac{5 \nu(6 \beta+7)}{28 \beta} .
$$

In the diffusion dominated limit (i.e. $P e_{S} \ll 1$ ) if we substitute $M=0$ in the presently obtained surfactant concentration $\Gamma=\Gamma^{(0)}+C a \Gamma^{(C a)}$ and $O(C a)$ drop shape correction $f^{(\mathrm{Ca})}$, they become similar to those obtained by Mandal et al. (2017b). In all these our calculations consistently capture the result of $N_{2}<0$ in the absence of electrical effects. This is commonly observed in polymeric solutions and has been reported by earlier studies Li \& Pozrikidis (1997).

(iii) Uncontaminated drop in combined shear flow and electric field:

In the limit $\beta \rightarrow 0$ the surface tension becomes uniform along the drop surface. For that situation, the present results e.g. the drop deformation, effective shear viscosity and normal stress differences in various orders of perturbation, match exactly with those of Mandal \& Chakraborty (2017b). When the electric field is applied along the direction of velocity gradient $\Phi_{t}=\pi / 2$, we find that in equation (37) the clean drop effective viscosity due to electric field $\left(\eta_{\mathrm{e}, \mathrm{clean}}^{(0)}\right)$ vanishes. A similar observation was also made in another reported work (Vlahovska, 2011). The electric field contribution, to the normal stress differences in the leading order (equation (38), are also in agreement with them.

Hence we obtained a reasonable confidence on the correctness of the theoretical analysis presented in this work.

\section{Appendix C. Effect of Mason number on surface tension}

In this section in figure $\mathrm{A}-1$ we delineate the effects of strength of electrical stresses relative to the hydrodynamic stresses, by varying the Mason number, define as $M=\frac{\epsilon_{c} \widetilde{E}_{\infty}^{2}}{\mu_{c} \widetilde{G}}$.

\section{Appendix D. Effect of $\beta$ and $k$ on surface concentration and surface tension}

Here in figure A-2, we explore the role of the surfactant characterization parameters, namely the elasticity number $\beta$ and physicochemical constant $k$, in altering the surfactant concentration and surface tension variation on the drop surface.

\section{Appendix E. Variation of interfacial velocity and electrical traction}

Here we exemplify the coupled consequence of charge convection and Marangoni stress on the flow field by showing the variation in the azimuthal components the drop velocity and electrical traction at the interface in figures $\mathrm{A}-3$. 


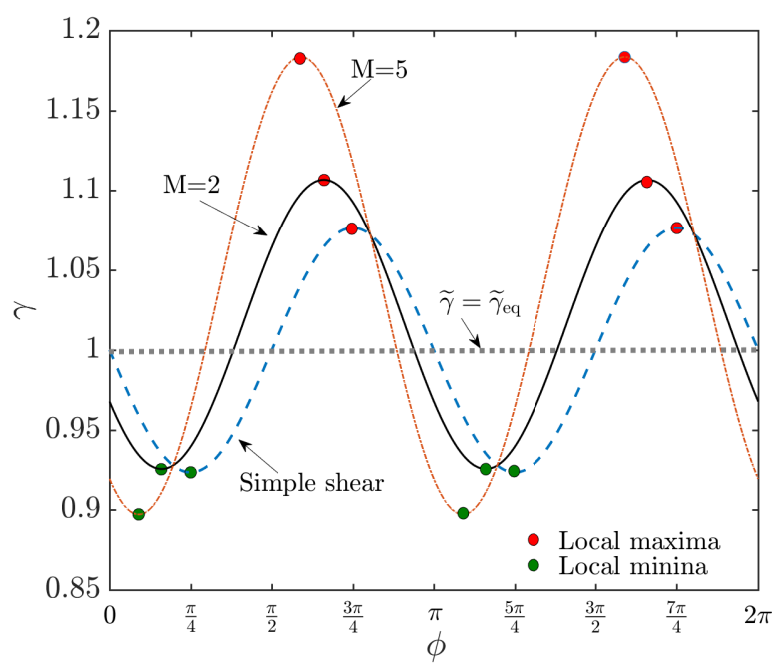

Figure A-1: Interfacial tension variation $(\gamma)$ in the $x-y$ plane for different Mason number $(M)$. Here $\beta=0.8, k=1$ and $\Phi_{t}=\pi / 2$. Other parameters are chosen following system-A.

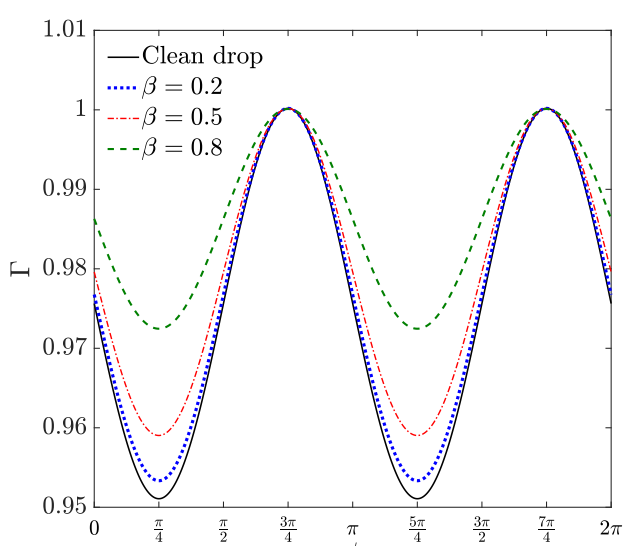

(a)

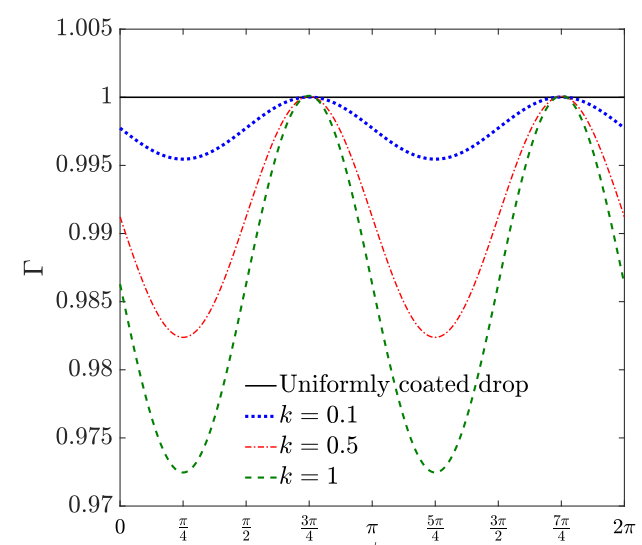

(c)

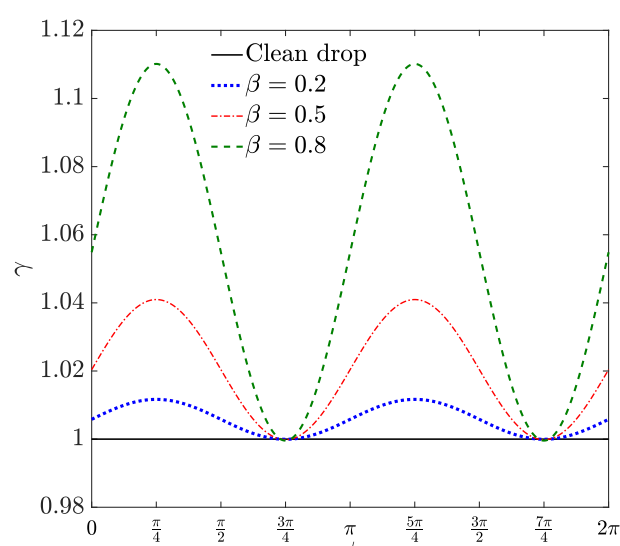

(b)

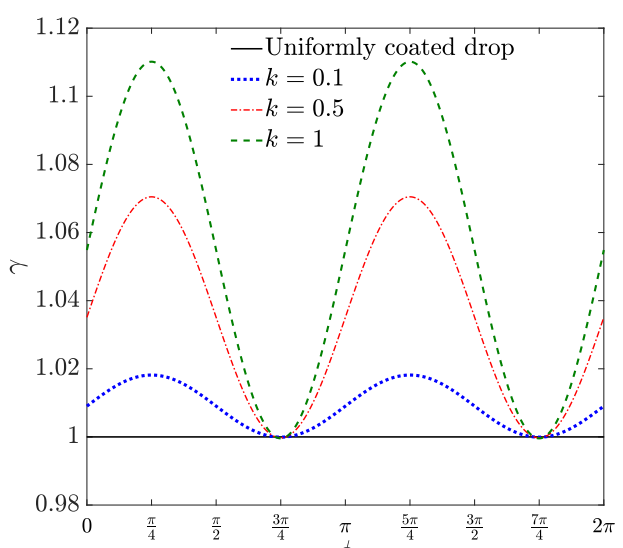

(d)

Figure A-2: Variation of surfactant concentration $\left(\Gamma=\Gamma^{(0)}+C a \Gamma^{(C a)}\right)$ and interfacial tension $\left(\gamma=\gamma^{(0)}+C a \gamma^{(C a)}\right)$ in the $x-y$ plane for different values of the parameters $\beta$ and $k$. In sub-figures (a) and (b), $k=1$ is chosen while $\beta=0.8$ is taken for (c) and (d). Here the tilt angle of the electric field is $\Phi_{t}=\pi / 4$, Mason number $M=2$ and other parameters are as per system-C. 


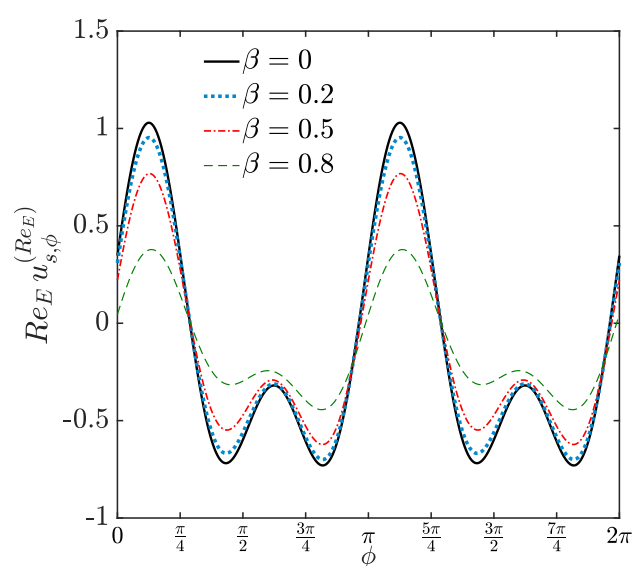

(a)

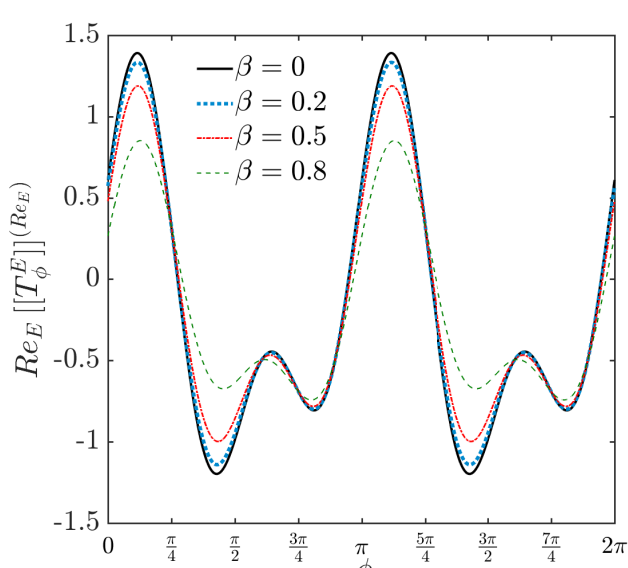

(b)

Figure A-3: Variation of $O\left(R e_{E}\right)$ correction to the surface velocity component, $R e_{E} u_{S, \phi}^{\left(R e_{E}\right)}$ and electrical traction component at the drop surface, $\left[\left[T_{\phi}\right]\right]^{\left(R e_{E}\right)}=\left.T_{\phi}^{E\left(R e_{E}\right)}\right|_{c}-\left.T_{\phi}^{E\left(R e_{E}\right)}\right|_{d}$ for different surface elasticity parameter, $\beta$. Here the demonstration os for system-C, while rest of the parameters are $M=2, R e_{E}=0.2, \Phi_{t}=\pi / 4$ and $k=1$.

\section{Appendix F. Surfactant effect on drop deformation}

In this section (figure A-4 we plotted the drop shapes for different electrohydrodynamic systems. Also the variation of deformation parameter $\mathcal{D}$ with the surface elasticity number $\beta$ is shown.

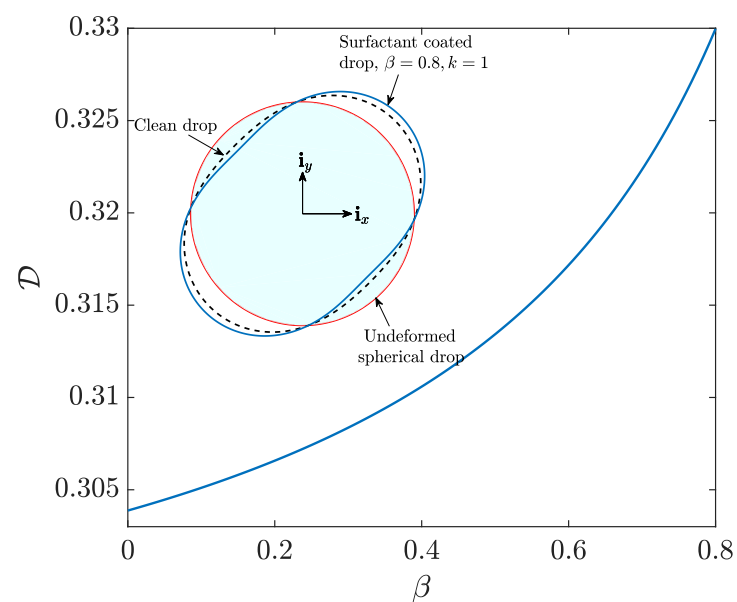

(a) System-B

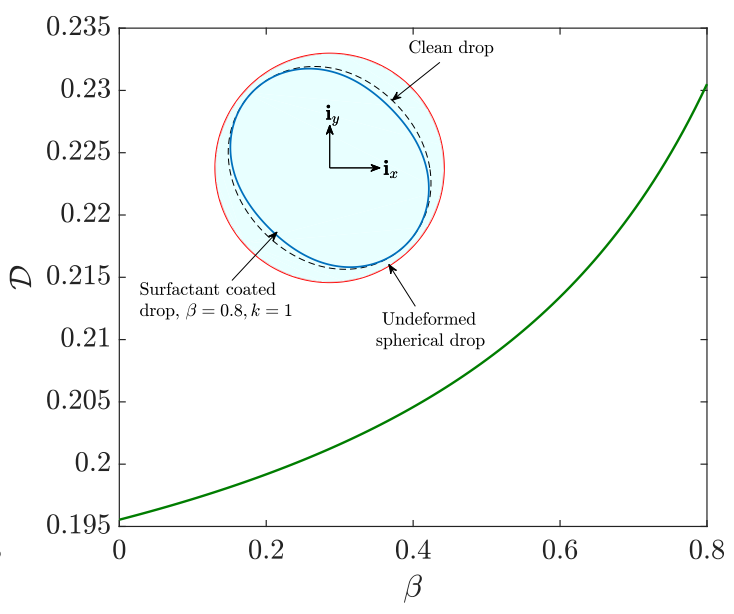

(b) System-C

Figure A-4: Effect of non-uniform surfactant distribution on the drop shape deformation in the plane of shear for systems B and C, respectively. The other parameters are $M=2, C a=$ $0.2, \Phi_{t}=\pi / 4$.

\section{References}

Allan, R. S. \& Mason, S. G. 1962 Particle behaviour in shear and electric fields. i. deformation and burst of fluid drops. Proc. R. Soc. A 267, 45. 
Anna, Shelley L., Bontoux, Nathalie \& Stone, Howard A. 2003 Formation of dispersions using flow focusing in microchannels. Applied Physics Letters 82 (3), 364-366.

Bandopadhyay, A., Mandal, S., Kishore, N. K. \& Chakraborty, S. 2016 Uniform electric-field-induced lateral migration of a sedimenting drop. J. Fluid Mech. 792 (2016), $553-589$.

Barnes, Howard A. 1994 Rheology of emulsions a review. Colloids and Surfaces A: Physicochemical and Engineering Aspects 91, 89 - 95, a selection of papers presented at the First World Congress on Emulsions.

Barths-Biesel, D. \& Acrivos, A. 1973 Deformation and burst of a liquid droplet freely suspended in a linear shear field. J. Fluid Mech. 61, 1.

BAtchelor, G. K. 1970 The stress system in a suspension of force-free particles. J. Fluid Mech. 41 (3), 545-570.

Bentley, B. J. \& Leal, L. G. 1986 An experimental investigation of drop deformation and breakup in steady, two-dimensional linear flows. J. Fluid Mech. 167, 241-283.

Bird, R. B., Armstrong, R. C. \& Hassager, O. 1987 Dynamics of polymeric liquids. vol. 1: Fluid mechanics .

Chan, P. C.-H. \& Leal, L. G. 1979 The motion of a deformable drop in a second-order fluid. J. Fluid Mech. 92 (01), 131--170.

Das, D. \& Saintillan, D. 2016 A nonlinear small-deformation theory for transient droplet electrohydrodynamics. J. Fluid Mech. 810, 225.

Eow, John S. \& Ghadiri, Mojtaba 2002 Electrostatic enhancement of coalescence of water droplets in oil: a review of the technology. Chemical Engineering Journal 85 (2), 357 - 368.

Ervik, Å., Penne, T. E., Helles $\varnothing$, S. M., Munkejord, S. T. \& Müller, B. 2018 Influence of surfactants on the electrohydrodynamic stretching of water drops in oil. Int. J. Multiphase Flow 98, 96-109.

Esmaeeli, Asghar \& Sharifi, Payam 2011 Transient electrohydrodynamics of a liquid drop. Physical Review E 84 (3), 036308.

FEnG, J. Q. 1999 Electrohydrodynamic behaviour of a drop subjected to a steady uniform electric field at finite electric Reynolds number. Proc. R. Soc. Lond. A 455 (1986), 2245-2269.

Fernndez, A. 2008 Response of an emulsion of leaky dielectric drops immersed in a simple shear flow: Drops less conductive than the suspending fluid. Phys. Fluids 20, 043304.

Fischer, P. \& ERni, P. 2007 Emulsion drops in external flow fields-the role of liquid interfaces. Curr. Opin. Colloid Interface Sci. 12 (4-5), 196-205.

Guazzelli, E. \& Morris, J. F. 2011 A physical introduction to suspension dynamics, , vol. 45. Cambridge University Press. 
Ha, Jong-Wook, Moon, Jun Hyuk \& Yang, Seung-Man 1999 Effect of nonionic surfactants on the electrorheology of emulsions. Korea-Australia Rheology Journal 11 (3), 241-246.

HA, J.-W. \& YANG, S.-M. 1995 Effects of surfactant on the deformation and stability of a drop in a viscous fluid in an electric field. J. Colloid Interface Sci. 175 (2), 369-385.

Ha, Jong-Wook \& YAng, SEung-Man 2000 Rheological responses of oil-in-oil emulsions in an electric field. Journal of Rheology 44 (2), 235-256.

Haber, S. \& Hetsroni, G. 1971 The dynamics of a deformable drop suspended in an unbounded Stokes flow. J. Fluid Mech. 49 (02), 257-277.

Haber, S. \& Hetsroni, G. 1972 Hydrodynamics of a drop submerged in an unbounded arbitrary velocity field in the presence of surfactants. Appl. Sci. Res. 25 (1), 215-233.

Haliburton, J. R., Kim, S. C., Clark, I. C., Sperling, R. A., Weitz, D. A. \& Abate, A. R. 2017 Efficient extraction of oil from droplet microfluidic emulsions. Biomicrofluidics 11 (3), 034111.

Happel, J \& BRenneR, H 1981 Low Reynolds number hydrodynamics. Springer.

Hu, Y. \& LIPS, A. 2003 Estimating surfactant surface coverage and decomposing its effect on drop deformation. Phys. Rev. Lett. 91, 044501.

JeOn, H. K. \& Macosko, C. W. 2003 Visualization of block copolymer distribution on a sheared drop. Polymer 44, 5381.

Kim, S \& Karila, S. J. 1991 Microhydrodynamics: Principles and Selected Applications. , Butterworth-Heinemann, Boston.

Lanauze, J. A., Walker, L. M. \& Khair, A. S 2015 Nonlinear electrohydrodynamics of slightly deformed oblate drops. J. Fluid Mech. 774, 245-266.

LEAL, L. G. 2007 Advanced transport phenomena: fluid mechanics and convective transport processes. Cambridge University Press.

Lequeux, F. 1998 Emulsion rheology. Curr. Opin. Colloid Interface Sci. 3 (4), 408-411.

Li, X. \& Pozrikidis, C. 1997 The effect of surfactants on drop deformation and on the rheology of dilute emulsions in Stokes flow. J. Fluid Mech. 341 (1997), 165-194.

Maehlmann, Stefan \& Papageorgiou, Demetrios T 2009 Numerical study of electric field effects on the deformation of two-dimensional liquid drops in simple shear flow at arbitrary reynolds number. Journal of Fluid Mechanics 626, 367-393.

Mandal, S., Bandopadhyay, A. \& Chakraborty, S. 2016 a Dielectrophoresis of a surfactant-laden viscous drop. Phys. Fluids 28 (6).

Mandal, S., Bandopadhyay, A. \& Chakraborty, S. $2016 b$ The effect of uniform electric field on the cross-stream migration of a drop in plane Poiseuille flow. J. Fluid Mech. 809 (2016), 726-774. 
Mandal, S., Chakrabarti, S. \& Chakraborty, S. 2017 a Effect of nonuniform electric field on the electrohydrodynamic motion of a drop in poiseuille flow. Physics of Fluids 29 (5), 052006 .

Mandal, S. \& Chakraborty, S. $2017 a$ Effect of uniform electric field on the drop deformation in simple shear flow and emulsion shear rheology. Physics of Fluids 29 (7), 072109.

Mandal, S. \& Chakraborty, S. $2017 b$ Uniform electric-field-induced non-newtonian rheology of a dilute suspension of deformable newtonian drops. Phys. Rev. Fluids 2, 093602.

Mandal, S., Das, S. \& Chakraborty, S. $2017 b$ Effect of marangoni stress on the bulk rheology of a dilute emulsion of surfactant-laden deformable droplets in linear flows. Phys. Rev. Fluids 2, 113604.

Melcher, J. R. \& TAYloR, G. I. 1969 Electrohydrodynamics: a review of the role of interfacial shear stresses. Annu. Rev. Fluid Mech. 1 (1), 111-146.

Milliken, W. J., Stone, H. A. \& Leal, L. G. 1993 The effect of surfactant on transient motion of newtonian drops. Phys. Fluids A 5, 69.

Na, Yang Ho, Aida, Kohei, Sakai, Ryosuke, Kakuchi, Toyoji \& Orihara, Hiroshi 2009 Response of shear stress to ac electric fields under steady shear flow in a dropletdispersed phase. Physical Review E 80 (6), 061803.

Nganguia, H., Young, Y. N., Vlahovska, P. M., BŁawzdziewcz, J., Zhang, J. \& Lin, H. 2013 Equilibrium electro-deformation of a surfactant-laden viscous drop. Phys. Fluids 25 (9).

Pak, O. S., Feng, J. \& Stone, H. A 2014 Viscous marangoni migration of a drop in a poiseuille flow at low surface péclet numbers. J. Fluid Mech. 753, 535-552.

Pan, X. D. \& McKinley, G. H. 1997 Characteristics of electrorheological responses in an emulsion system. J. Colloid Interface Sci. 195, 101.

PAWAR, Y. \& SteBe, K. J. 1996 Marangoni effects on drop deformation in an extensional flow: The role of surfactant physical chemistry. i. insoluble surfactants. Phys. Fluids 8 (7), $1738-1751$.

Poddar, A., Mandal, S., Bandopadhyay, A. \& Chakraborty, S. 2018 Sedimentation of a surfactant-laden drop under the influence of an electric field. J. Fluid Mech. 849, 277311.

Puyvelde, Peter Van, Velankar, Sachin \& Moldenaers, Paula 2001 Rheology and morphology of compatibilized polymer blends. Current Opinion in Colloid \& Interface Science 6 (5), $457-463$.

RAllison, J. M. 1984 The deformation of small viscous drops and bubbles in shear flows. Annu. Rev. Fluid Mech. 16, 45. 
Salipante, P. F. \& Vlahovska, P. M. 2010 Electrohydrodynamics of drops in strong uniform dc electric fields. Phys. Fluids 22 (11), 112110.

SAVILle, D. A. 1997 Electrohydrodynamics: the taylor-melcher leaky dielectric model. Annu. Rev. Fluid Mech. 29 (1), 27-64.

Schowalter, W. R., Chaffey, C. E. \& Brenner, H. 1968 Rheological behavior of a dilute emulsion. Journal of colloid and interface science 26 (2), 152-160.

Stone, H. A. 1990 A simple derivation of the time-dependent convective-diffusion equation for surfactant transport along a deforming interface. Phys. Fluids A 2, 111.

Stone, H. A. \& Leal, L. G. 1990 The effects of surfactants on drop deformation and breakup. J. Fluid Mech. 220, 161.

TAYLOR, G. 1966 Studies in electrohydrodynamics. i. the circulation produced in a drop by an electric field. Proc. R. Soc. Lond. A 291 (1425), 159-166.

TAYlor, G. I. 1932 The viscosity of a fluid containing small drops of another fluid. Proc. $R$. Soc. Lond. A 138 (834), 41-48.

Teigen, K. \& Munkejord, S. T. 2010 Influence of surfactant on drop deformation in an electric field. Phys. Fluids 22 (11).

ThAOKAR, RM 2012 Dielectrophoresis and deformation of a liquid drop in a non-uniform, axisymmetric ac electric field. The European Physical Journal E 35 (8), 76.

Tsukada, T., Katayama, T., Ito, Y. \& Hozawa, M. 1993 Theoretical and Experimental Studies of Circulations Inside and Outside a Deformed Drop under a Uniform Electric Field. J. Chem. Eng. Jpn. 26 (6), 698-703.

Tucker, C. \& Moldenaers, P. 2002 Microstructural evolution in polymer blends. Annu. Rev. Fluid Mech. 34, 177.

Valkovska, D. S. \& Danov, K. D. 2000 Determination of bulk and surface diffusion coefficients from experimental data for thin liquid film drainage. Journal of colloid and interface science 223 (2), 314-316.

Velankar, S., Van Puyvelde, P., Mewis, J. \& Moldenaers, P. 2004 Steady shear rheological properties of model compatibilized blends. J. Rheol. 48, 725.

Velankar, S., Van Pyuvede, P., Mewis, J. \& Moldenaers, P. 2001 Effect of compatibilization on the breakup of polymeric drops in shear flow. J. Rheol. 45, 1007.

Vlahovska, P. M. 2011 On the rheology of a dilute emulsion in a uniform electric field. $J$. Fluid Mech. 670, 481-503.

Vlahovska, P. M. 2019 Electrohydrodynamics of drops and vesicles. Annu. Rev. Fluid Mech. 51 (1), null. 
Vlahovska, P. M., BŁawzdziewicz, J. \& Loewenberg, M. 2009 Small-deformation theory for a surfactant-covered drop in linear flows. J. Fluid Mech. 624, 293-337.

Vlahovska, P. M., Loewenberg, M. \& Blawzdziewicz, J. 2005 Deformation of a surfactant-covered drop in a linear flow. Physics of Fluids 17 (10), 103103.

Xu, J. J., Li, Z., Lowengrub, J. \& ZhaO, H. 2006 A level-set method for interfacial flows with surfactant. J. Comput. Phys. 212 (2), 590-616.

Xu, X. \& Homsy, G. M. 2006 The settling velocity and shape distortion of drops in a uniform electric field. J. Fluid Mech. 564, 395.

YARIV, E. \& Almog, Y. 2016 The effect of surface-charge convection on the settling velocity of spherical drops in a uniform electric field. J. Fluid Mech. 797, 536-548. 